Algebraic 83 Geometric $\mathcal{T}_{\text {opology }}$

Volume 5 (2005) 653-690

Published: 5 July 2005

ATG

\title{
Differentials in the homological homotopy fixed point spectral sequence
}

\author{
Robert R. Bruner \\ JoHn Rognes
}

\begin{abstract}
We analyze in homological terms the homotopy fixed point spectrum of a $\mathbb{T}$-equivariant commutative $S$-algebra $R$. There is a homological homotopy fixed point spectral sequence with $E_{s, t}^{2}=H_{g p}^{-s}\left(\mathbb{T} ; H_{t}\left(R ; \mathbb{F}_{p}\right)\right)$, converging conditionally to the continuous homology $H_{s+t}^{c}\left(R^{h \mathbb{T}} ; \mathbb{F}_{p}\right)$ of the homotopy fixed point spectrum. We show that there are Dyer-Lashof operations $\beta^{\epsilon} Q^{i}$ acting on this algebra spectral sequence, and that its differentials are completely determined by those originating on the vertical axis. More surprisingly, we show that for each class $x$ in the $E^{2 r}$-term of the spectral sequence there are $2 r$ other classes in the $E^{2 r}$-term (obtained mostly by Dyer-Lashof operations on $x$ ) that are infinite cycles, i.e., survive to the $E^{\infty}$-term. We apply this to completely determine the differentials in the homological homotopy fixed point spectral sequences for the topological Hochschild homology spectra $R=T H H(B)$ of many $S$-algebras, including $B=M U, B P, k u, k o$ and $t m f$. Similar results apply for all finite subgroups $C \subset \mathbb{T}$, and for the Tate- and homotopy orbit spectral sequences. This work is part of a homological approach to calculating topological cyclic homology and algebraic $K$-theory of commutative $S$-algebras.
\end{abstract}

AMS Classification 19D55, 55S12, 55T05; 55P43, 55P91

Keywords Homotopy fixed points, Tate spectrum, homotopy orbits, commutative $S$-algebra, Dyer-Lashof operations, differentials, topological Hochschild homology, topological cyclic homology, algebraic $K$-theory

\section{Introduction}

In this paper we study a homological version of the homotopy fixed point spectral sequence of an equivariant spectrum, with emphasis on the interaction between differentials in the spectral sequence and strictly commutative products in the equivariant spectrum. We begin by describing the intended application of this study. 
Consider a connective $S$-algebra $B$, such as the sphere spectrum $S$, the complex cobordism spectrum $M U$ or the Eilenberg-Mac Lane spectrum $H \mathbb{Z}$ of the integers, in either of the current frameworks [BHM93, [EKMM97, HSS00, MMSS01 for structured ring spectra. The algebraic $K$-theory spectrum $K(B)$ can, according to the main theorem of Du97, be very well approximated by the topological cyclic homology spectrum $T C(B)$ of BHM93. The latter is constructed from the $\mathbb{T}$-equivariant topological Hochschild homology spectrum $X=T H H(B)$, where $\mathbb{T}$ is the circle group, as a homotopy limit of the fixed point spectra $X^{C}$, suitably indexed over finite cyclic subgroups $C \subset \mathbb{T}$.

These fixed point spectra are in turn often well approximated, via canonical maps $\gamma: X^{C} \rightarrow X^{h C}$, by homotopy fixed point spectra $X^{h C}=F\left(E C_{+}, X\right)^{C}$. In principle, the homotopy groups of the latter can be computed by the homotopical homotopy fixed point spectral sequence

$$
E_{s, t}^{2}=H_{g p}^{-s}\left(C ; \pi_{t}(X)\right) \Longrightarrow \pi_{s+t}\left(X^{h C}\right),
$$

which is derived by applying homotopy groups to the tower of fibrations, with limit $X^{h C}$, that arises from the equivariant skeleton filtration on the free contractible $C$-space $E C$.

Such computations presume a detailed knowledge of the homotopy groups $\pi_{*}(X)$ of the $\mathbb{T}$-equivariant spectrum in question. For example, the papers HM03 and AuR02 deal with the cases when $B$ is the valuation ring $H \mathcal{O}_{K}$ in a local number field $K$ and the Adams summand $\ell_{p}$ in $p$-complete connective topological $K$-theory $k u_{p}$, respectively. In most other cases the spectral sequence (1.1) cannot be calculated, because the homotopy groups $\pi_{*}(X)$ are not sufficiently well known.

It happens more frequently in stable homotopy theory that we are familiar with the homology groups $H_{*}\left(X ; \mathbb{F}_{p}\right)$. Applying mod $p$ homology, rather than homotopy, to the tower of fibrations that approximates $X^{h C}$ leads to a homological homotopy fixed point spectral sequence

$$
E_{s, t}^{2}=H_{g p}^{-s}\left(C ; H_{t}\left(X ; \mathbb{F}_{p}\right)\right) \Longrightarrow H_{s+t}^{c}\left(X^{h C} ; \mathbb{F}_{p}\right) .
$$

This spectral sequence converges conditionally Bo99 to the (inverse) limit of the resulting tower in homology, which is not $H_{*}\left(X^{h C} ; \mathbb{F}_{p}\right)$, but a "continuous" version $H_{*}^{c}\left(X^{h C} ; \mathbb{F}_{p}\right)$ of it, for homology does not usually commute with limits.

This continuous homology, considered as a comodule over the dual Steenrod algebra $A_{*}$ Mi58, is nonetheless a powerful invariant of $X^{h C}$. In particular, when $X$ is bounded below and of finite type there is a strongly convergent spectral sequence

$$
E_{2}^{s, t}=\operatorname{Ext}_{A_{*}}^{s, t}\left(\mathbb{F}_{p}, H_{*}^{c}\left(X^{h C} ; \mathbb{F}_{p}\right)\right) \Longrightarrow \pi_{t-s}\left(X^{h C}\right)_{p}^{\wedge}
$$


which can be obtained as an inverse limit of Adams spectral sequences CMP87, 7.1]. Hence the continuous homology does in some sense determine the $p$-adic homotopy type of $X^{h C}$.

A form of the spectral sequence (1.3) was most notably applied in the proofs by W. H. Lin LDMA80 and J. Gunawardena AGM85] of the Segal conjecture for cyclic groups of prime order. The conjecture corresponds to the special case of the discussion above when $B=S$ is the sphere spectrum, so $X=T H H(S)=$ $S$ is the $\mathbb{T}$-equivariant sphere spectrum, which is split [LMS86, II.8] so that $X^{h C} \simeq F\left(B C_{+}, S\right)=D\left(B C_{+}\right)$. The proven Segal conjecture Ca84 then tells us that for each $p$-group $C$ the comparison map $\gamma: X^{C} \rightarrow X^{h C}$ is a $p$-adic equivalence. The proof of the general (cyclic) case is by reduction to the initial case when $C=C_{p}$ is of prime order, and therefore relies on the theorems of Lin and Gunawardena cited above. In this case, of course, we do not know $\pi_{*}(X)=\pi_{*}(S)$ sufficiently well, but $H_{*}\left(X ; \mathbb{F}_{p}\right)=\mathbb{F}_{p}$ is particularly simple. The proof of the theorems of Lin and Gunawardena now amounts to showing that although the natural homomorphism $\gamma_{*}: H_{*}\left(X^{C} ; \mathbb{F}_{p}\right) \rightarrow H_{*}^{c}\left(X^{h C} ; \mathbb{F}_{p}\right)$ of $A_{*}$-comodules is not in itself an isomorphism, it does induce an isomorphism of $E_{2}$-terms upon applying the functor $\operatorname{Ext}_{A_{*}}^{* *}\left(\mathbb{F}_{p},-\right)$.

Returning to the general situation, we are therefore interested in studying (i) the differentials in the homological homotopy fixed point spectral sequence (1.2) above, and (ii) the $A_{*}$-comodule extension questions relating its $E^{\infty}$-term to the abutment $H_{*}^{c}\left(X^{h C} ; \mathbb{F}_{p}\right)$. There will in general be non-trivial differentials in (1.2), but our main Theorem 1.2 below provides a very general and useful vanishing result, as is illustrated by the examples in Section 6. The identification of the $A_{*}$-comodule structure on the abutment plays a crucial role already in the case $X=S$, but requires further study beyond that given here, and will be presented in the forthcoming Ph.D. thesis of Sverre Lunøe-Nielsen [L-N].

Now suppose that $B$ is a commutative $S$-algebra, in either one of the structured categories listed at the outset. Then $X=T H H(B)$ can be constructed as a $\mathbb{T}$-equivariant commutative $S$-algebra, which we hereafter denote $R=X$, in the naive sense of a commutative $S$-algebra with a continuous $\mathbb{T}$-action through commutative $S$-algebra maps. Starting at this point we need to be in a technical framework where naively $\mathbb{T}$-equivariant commutative $S$-algebras $R$ make sense, and either of the $S$-modules of [EKMM97, the orthogonal spectra of [MMSS01, the equivariant orthogonal spectra of MM02 or the topological version of the symmetric spectra of [HSS00] will do. To be concrete, we can follow [EKMM97, Ch. IX].

Then the tower of fibrations with limit $R^{h C}=X^{h C}$ is one of commutative $S$ algebras. It follows that there are Dyer-Lashof operations acting on the spectral 
sequence (1.2) in this case, somewhat analogously to the action by Steenrod operations in the Adams spectral sequence of a commutative $S$-algebra BMMS86 Ch. IV]. In the latter case there are very interesting relations between the Adams differentials and the Steenrod operations, which propagate early differentials to higher degrees. The initial motivation for the present article was to determine the analogous interaction between the differentials and the Dyer-Lashof operations in the homological homotopy fixed point spectral sequence of a commutative $S$-algebra. However, the analogy with the behavior of differentials in the Adams spectral sequence is more apparent than real, suggesting neither the survival to $E^{\infty}$ of some classes, nor the method of proof. In particular, there is no analog in the Adams spectral sequence of our main vanishing result, Theorem 1.2

For each finite subgroup $C \subset \mathbb{T}$ the homological spectral sequence for $R^{h C}$ is an algebra over the corresponding homological spectral sequence for $R^{h \mathbb{T}}$, as outlined in Section 7, so it will suffice for us to consider the circle homotopy fixed points $R^{h \mathbb{T}}$ and the case $C=\mathbb{T}$ of the spectral sequence (1.2). Our first results in Sections 2-4 can then be summarized as follows.

Theorem 1.1 (a) Let $R$ be a $\mathbb{T}$-equivariant commutative $S$-algebra. Then there is a natural $A_{*}$-comodule algebra spectral sequence

$$
E_{* *}^{2}(R)=H_{g p}^{-*}\left(\mathbb{T} ; H_{*}\left(R ; \mathbb{F}_{p}\right)\right)=P(y) \otimes H_{*}\left(R ; \mathbb{F}_{p}\right)
$$

with $y$ in bidegree $(-2,0)$, converging conditionally to the continuous homology

$$
H_{*}^{c}\left(R^{h \mathbb{T}} ; \mathbb{F}_{p}\right)=\lim _{n} H_{*}\left(F\left(S\left(\mathbb{C}^{n}\right)_{+}, R\right)^{\mathbb{T}} ; \mathbb{F}_{p}\right)
$$

of the homotopy fixed point spectrum $R^{h \mathbb{T}}=F\left(E \mathbb{T}_{+}, R\right)^{\mathbb{T}}$.

(b) There are natural Dyer-Lashof operations $\beta^{\epsilon} Q^{i}$ acting vertically on this homological homotopy fixed point spectral sequence. For each element $x \in$ $E_{0, t}^{2 r} \subset H_{t}\left(R ; \mathbb{F}_{p}\right)$ we have the relation

$$
d^{2 r}\left(\beta^{\epsilon} Q^{i}(x)\right)=\beta^{\epsilon} Q^{i}\left(d^{2 r}(x)\right)
$$

for every integer $i$ and $\epsilon \in\{0,1\}$. If $d^{2 r}(x)=y^{r} \cdot \delta x$ with $\delta x \in H_{t+2 r-1}\left(R ; \mathbb{F}_{p}\right)$, then the right hand side $\beta^{\epsilon} Q^{i}\left(d^{2 r}(x)\right)$ is defined to be $y^{r} \cdot \beta^{\epsilon} Q^{i}(\delta x)$.

(c) The classes $y^{n}$ are infinite cycles, so the differentials from the vertical axis $E_{0, *}^{2 r}$ propagate to each column by the relation

$$
d^{2 r}\left(y^{n} \cdot x\right)=y^{n} \cdot d^{2 r}(x)
$$

for every $x \in E_{0, *}^{2 r}, 2 r \geq 2, n \geq 0$. Hence there are isomorphisms $E_{* *}^{2 r} \equiv P(y) \otimes$ $E_{0, *}^{2 r}$ for all $2 r \geq 2$, modulo possible $y$-torsion in filtrations $-2 r+4 \leq s \leq 0$. 
For proofs, see Propositions 2.1 4.1 and 4.2, and Lemma 4.3. The key idea is to identify the differentials in the homological homotopy fixed point spectral sequence as obstructions to extending equivariant maps, as explained in Section 3. Note that the spectral sequence is concentrated in even columns, so all differentials of odd length ( $d^{r}$ with $r$ odd) must vanish.

Our main theorem is the following vanishing result for differentials.

Theorem 1.2 Let $R$ be a $\mathbb{T}$-equivariant commutative $S$-algebra, suppose that $x \in H_{t}\left(R ; \mathbb{F}_{p}\right)$ survives to the $E^{2 r}$-term $E_{0, t}^{2 r} \subset H_{t}\left(R ; \mathbb{F}_{p}\right)$ of the homological homotopy fixed point spectral sequence for $R$, and write $d^{2 r}(x)=y^{r} \cdot \delta x$.

(a) For $p=2$, the $2 r$ classes

$$
x^{2}=Q^{t}(x), Q^{t+1}(x), \ldots, Q^{t+2 r-2}(x) \text { and } Q^{t+2 r-1}(x)+x \delta x
$$

all survive to the $E^{\infty}$-term, i.e., are infinite cycles.

(b) For $p$ odd and $t=2 m$ even, the $2 r$ classes

$$
x^{p}=Q^{m}(x), \beta Q^{m+1}(x), \ldots, Q^{m+r-1}(x) \text { and } x^{p-1} \delta x
$$

all survive to the $E^{\infty}$-term, i.e., are infinite cycles.

(c) For $p$ odd and $t=2 m-1$ odd, the $2 r$ classes

$$
\beta Q^{m}(x), Q^{m}(x), \ldots, \beta Q^{m+r-1}(x) \quad \text { and } \quad Q^{m+r-1}(x)-x(\delta x)^{p-1}
$$

all survive to the $E^{\infty}$-term, i.e., are infinite cycles.

This will be proved in Section 5 as Theorem 5.1. There are similar extensions of our results to the Tate constructions $R^{t C}=\left[\widetilde{E C} \wedge F\left(E C_{+}, R\right)\right]^{C}$ and homotopy orbit spectra $R_{h C}=E C_{+} \wedge_{C} R$, but to keep the exposition simple these are also only discussed in Section 7 .

Remark 1.3 In more detail, in the three cases of the theorem the infinite cycles can be listed as follows:

(a) $x^{2}=Q^{t}(x), Q^{i}(x)$ for $t+1 \leq i \leq t+2 r-2$ and $Q^{t+2 r-1}(x)+x \delta x$

(b) $x^{p}=Q^{m}(x), \beta^{\epsilon} Q^{i}(x)$ for $m+1 \leq i \leq m+r-1, \epsilon \in\{0,1\}$ and $x^{p-1} \delta x$;

(c) $\beta^{\epsilon} Q^{i}(x)$ for $m \leq i \leq m+r-2, \epsilon \in\{0,1\}, \beta Q^{m+r-1}(x)$ and $Q^{m+r-1}(x)-$ $x(\delta x)^{p-1}$.

In particular, for any element $x \in H_{t}\left(R ; \mathbb{F}_{p}\right)$, with $d^{2}(x)=y \cdot \delta x$, there are two infinite cycles 
(a) $x^{2}=Q^{t}(x)$ and $Q^{t+1}(x)+x \delta x$ when $p=2$,

(b) $x^{p}=Q^{m}(x)$ and $x^{p-1} \delta x$ when $p$ is odd and $t=2 m$, and

(c) $\beta Q^{m}(x)$ and $Q^{m}(x)-x(\delta x)^{p-1}$ when $p$ is odd and $t=2 m-1$.

As applications of our main results, we turn in Section 6 to the study of the algebraic $K$-theory spectrum $K(M U)$, interpolating between $K(S)$ (which is Waldhausen's $A(*)$, related to high dimensional geometric topology) and $K(\mathbb{Z})$ (which relates to the Vandiver and Leopoldt conjectures, and other number theory), by the methods of topological cyclic homology. Hence we must study the fixed- and homotopy fixed point spectra of the commutative $S$-algebra $R=T H H(M U)$, for various subgroups $C$ of the circle group $\mathbb{T}$. It is known that $H_{*}\left(M U ; \mathbb{F}_{p}\right)=P\left(b_{k} \mid k \geq 1\right)$, where $P(-)$ denotes the polynomial algebra over $\mathbb{F}_{p}$ and $\left|b_{k}\right|=2 k$, from which it follows (MS93, 4.3] or [CS]) that $H_{*}\left(T H H(M U) ; \mathbb{F}_{p}\right)=H_{*}\left(M U ; \mathbb{F}_{p}\right) \otimes E\left(\sigma b_{k} \mid k \geq 1\right)$, where $E(-)$ denotes the exterior algebra over $\mathbb{F}_{p}$ and $\sigma: H_{*}\left(R ; \mathbb{F}_{p}\right) \rightarrow H_{*+1}\left(R ; \mathbb{F}_{p}\right)$ is the degree +1 operator induced by the circle action. Hence the homological homotopy fixed point spectral sequence for $T H H(M U)^{h T}$ begins

$$
E_{* *}^{2}=P(y) \otimes P\left(b_{k} \mid k \geq 1\right) \otimes E\left(\sigma b_{k} \mid k \geq 1\right) .
$$

There are differentials $d^{2}\left(b_{k}\right)=y \cdot \sigma b_{k}$ for all $k \geq 1$, so by our Theorem 1.1]

$$
E_{* *}^{4}=P(y) \otimes P\left(b_{k}^{p} \mid k \geq 1\right) \otimes E\left(b_{k}^{p-1} \sigma b_{k} \mid k \geq 1\right)
$$

plus some classes (the image of $\sigma$ ) in filtration $s=0$. By our Theorem 1.2 the spectral sequence collapses completely at the $E^{4}$-term, so that

$$
H_{*}^{c}\left(T H H(M U)^{h \mathbb{T}} ; \mathbb{F}_{p}\right)=P(y) \otimes P\left(b_{k}^{p} \mid k \geq 1\right) \otimes E\left(b_{k}^{p-1} \sigma b_{k} \mid k \geq 1\right)
$$

plus some classes in filtration zero, as an algebra. The identification of the $A_{*}$ comodule extensions remains, for which we refer to the cited Ph.D. thesis $\mathrm{L}-\mathrm{N}$. This provides the input for the inverse limit of Adams spectral sequences (1.3) converging to $\pi_{*}\left(T H H(M U)^{h \mathbb{T}}\right)_{p}^{\wedge}$, which approximates the topological version $T F(M U)$ of negative cyclic homology, and which determines the topological cyclic homology of $M U$ by a fiber sequence

$$
T C(M U) \stackrel{\pi}{\longrightarrow} T F(M U) \stackrel{R-1}{\longrightarrow} T F(M U) .
$$

The fiber of the cyclotomic trace map $K(M U) \rightarrow T C(M U)$ is equivalent to that of $K(\mathbb{Z}) \rightarrow T C(\mathbb{Z})$, by [Du97, which now is quite well known [Ro02, Ro03. Our theorem therefore provides a key input to the computation of $K(M U)$. See Theorem 6.4(a). 
Similar applications are given for the connective Johnson-Wilson spectra $B=$ $B P\langle n\rangle$, for $p$ and $n$ such that these are commutative $S$-algebras, and the (higher real) commutative $S$-algebras $B=k o$ and $\operatorname{tmf}$ for $p=2$. See Section 6. Lastly, we can also show the collapse at the $E^{4}$-term of the homological homotopy fixed point spectral sequence for $R=T H H(B P)$, where $B P$ is the $p$-local Brown-Peterson $S$-algebra BJ02, without making the (presently uncertain) assumption that $B P$ can be realized as a commutative $S$-algebra. See Theorem 6.4(b). This is possible by the homological approach, since the split surjection $H_{*}\left(M U ; \mathbb{F}_{p}\right) \rightarrow H_{*}\left(B P ; \mathbb{F}_{p}\right)$ prevails throughout the homological spectral sequences.

\section{A homological spectral sequence}

In this section we construct the homological homotopy fixed point spectral sequence for a spectrum with a circle action.

Let $\mathbb{T} \subset \mathbb{C}^{*}$ be the circle group. As our concrete model for a free contractible $\mathbb{T}$-CW complex $E \mathbb{T}$ we take the unit sphere $S\left(\mathbb{C}^{\infty}\right) \subset \mathbb{C}^{\infty}$ with the usual coordinatewise action by $\mathbb{T}$. It has one $\mathbb{T}$-equivariant cell in each even nonnegative dimension $2 n \geq 0$. The equivariant $2 n$-skeleton is the odd $(2 n+1)$ sphere $E \mathbb{T}^{(2 n)}=S\left(\mathbb{C}^{n+1}\right)$, which is obtained from the equivariant $(2 n-2)$ skeleton $E \mathbb{T}^{(2 n-2)}=S\left(\mathbb{C}^{n}\right)$ by attaching a free $\mathbb{T}$-equivariant $2 n$-cell $\mathbb{T} \times D^{2 n}$ along the group action map

$$
\alpha: \mathbb{T} \times \partial D^{2 n} \rightarrow S\left(\mathbb{C}^{n}\right) .
$$

This map is $\mathbb{T}$-equivariant when we give $\partial D^{2 n}$ the trivial $\mathbb{T}$-action and $S\left(\mathbb{C}^{n}\right)$ the free $\mathbb{T}$-action. Hence there is a $\mathbb{T}$-equivariant filtration

$$
\emptyset \subset S(\mathbb{C}) \subset \cdots \subset S\left(\mathbb{C}^{n}\right) \subset S\left(\mathbb{C}^{n+1}\right) \subset \cdots
$$

with colimit $E \mathbb{T}$, and $\mathbb{T}$-equivariant cofiber sequences

$$
S\left(\mathbb{C}^{n}\right) \rightarrow S\left(\mathbb{C}^{n+1}\right) \rightarrow \mathbb{T}_{+} \wedge S^{2 n}
$$

for $n \geq 0$. Here $\mathbb{T}$ acts trivially on $S^{2 n}=D^{2 n} / \partial D^{2 n}$.

Let $X$ be any spectrum with $\mathbb{T}$-action, i.e., a naively $\mathbb{T}$-equivariant spectrum. The homotopy fixed point spectrum of $X$ is defined as the mapping spectrum

$$
X^{h \mathbb{T}}=F\left(E \mathbb{T}_{+}, X\right)^{\mathbb{T}}
$$


of $\mathbb{T}$-equivariant based maps from $E \mathbb{T}_{+}$to $X$. Here $E \mathbb{T}_{+}$should be interpreted as the unreduced suspension spectrum of $E \mathbb{T}$. The $\mathbb{T}$-equivariant filtration of $E \mathbb{T}=S\left(\mathbb{C}^{\infty}\right)$ induces a tower of fibrations

$$
\cdots \rightarrow F\left(S\left(\mathbb{C}^{n+1}\right)_{+}, X\right)^{\mathbb{T}} \rightarrow F\left(S\left(\mathbb{C}^{n}\right)_{+}, X\right)^{\mathbb{T}} \rightarrow \cdots \rightarrow F\left(S(\mathbb{C})_{+}, X\right)^{\mathbb{T}} \rightarrow *
$$

with the homotopy fixed point spectrum as its limit

$$
X^{h \mathbb{T}}=\lim _{n} F\left(S\left(\mathbb{C}^{n}\right)_{+}, X\right)^{\mathbb{T}},
$$

weakly equivalent to the homotopy limit. The cofiber sequences above induce cofiber sequences of spectra

$$
\Sigma^{-2 n} X=F\left(\mathbb{T}_{+} \wedge S^{2 n}, X\right)^{\mathbb{T}} \stackrel{k}{\rightarrow} F\left(S\left(\mathbb{C}^{n+1}\right)_{+}, X\right)^{\mathbb{T}} \stackrel{i}{\rightarrow} F\left(S\left(\mathbb{C}^{n}\right)_{+}, X\right)^{\mathbb{T}}
$$

for each $n \geq 0$. We place $F\left(E \mathbb{T}_{+}^{(-s-1)}, X\right)^{\mathbb{T}}$ in filtration $s$, for each $s \in \mathbb{Z}$, and obtain a chain of cofiber sequences of spectra:

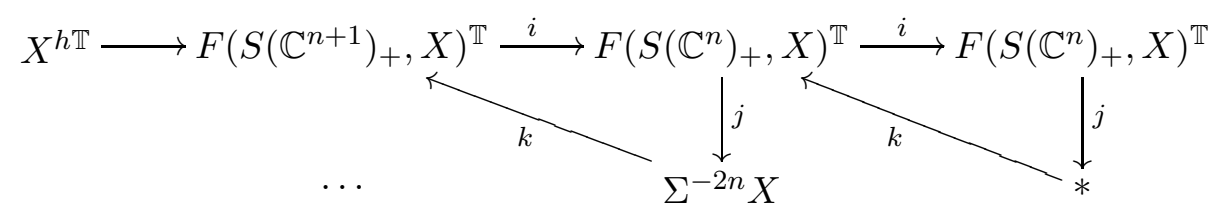

Here the filtrations $s=-2 n-1,-2 n$ and $-2 n+1$ are displayed, and the vertical maps labeled $j$ are of degree -1 .

Now apply mod $p$ homology $H_{*}\left(-; \mathbb{F}_{p}\right)$ to this chain of cofiber sequences, to obtain a homologically indexed unrolled exact couple [Bo99, 0.1] of graded $A_{*-}$ comodules

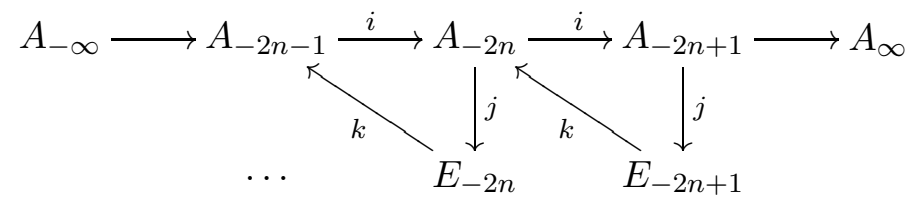

with

$$
A_{s, t}=H_{s+t}\left(F\left(E \mathbb{T}_{+}^{(-s-1)}, X\right)^{\mathbb{T}} ; \mathbb{F}_{p}\right)
$$

equal to $H_{s+t}\left(F\left(S\left(\mathbb{C}^{n}\right)_{+}, X\right)^{\mathbb{T}} ; \mathbb{F}_{p}\right)$ for $s=-2 n$ and $s=-2 n+1$, and

$$
E_{s, t}=H_{s+t}\left(F\left(E \mathbb{T}^{(-s)} / E \mathbb{T}^{(-s-1)}, X\right)^{\mathbb{T}} ; \mathbb{F}_{p}\right)
$$

equal to $H_{s+t}\left(\Sigma^{-2 n} X ; \mathbb{F}_{p}\right) \cong H_{t}\left(X ; \mathbb{F}_{p}\right)$ for $s=-2 n \leq 0$, and zero otherwise. We write $y^{n} \cdot x \in E_{-2 n, t}$ for the class that corresponds to $x \in H_{t}\left(X ; \mathbb{F}_{p}\right)$ under this canonical suspension isomorphism. 
The associated spectral sequence $E^{r}=E^{r}(X)$ with

$$
E_{s, t}^{1}=E_{s, t} \cong \begin{cases}H_{t}\left(X ; \mathbb{F}_{p}\right) & \text { for } s=-2 n \leq 0 \\ 0 & \text { otherwise }\end{cases}
$$

is by definition the homological homotopy fixed point spectral sequence for the $\mathbb{T}$-equivariant spectrum $X$. The $E^{1}$-term is concentrated in the non-positive even columns, so $d^{1}=0$ and $E^{1}=E^{2}$.

Alternatively, there is (the finite part of) a Cartan-Eilenberg system CE56, XV.7], with graded groups

$$
H(a, b)=H_{*}\left(F\left(E \mathbb{T}^{(b-1)} / E \mathbb{T}^{(a-1)}, X\right)^{\mathbb{T}} ; \mathbb{F}_{p}\right)
$$

for all integers $a \leq b$, which via $E_{s}^{1}=H(-s,-s+1)$ gives rise to the same spectral sequence as the unrolled exact couple above. Also define $H(a, \infty)=$ $\lim _{b} H(a, b)$, so that $H(0, \infty)=H_{*}^{c}\left(X^{h \mathbb{T}} ; \mathbb{F}_{p}\right)$. We shall refer to this formalism when discussing products in Section 4.

Since $A_{s}=0$ for $s \geq 0$ we trivially have $A_{\infty}=\operatorname{colim}_{s} A_{s}=0$. Therefore the associated spectral sequence is conditionally convergent, by [Bo99, 5.10], in this case to the limit $A_{-\infty}=\lim _{s} A_{s}$. Indexing the limit system by $n$ in place of $s$, the abutment can be written as

$$
H_{*}^{c}\left(X^{h \mathbb{T}} ; \mathbb{F}_{p}\right)=\lim _{n} H_{*}\left(F\left(S\left(\mathbb{C}^{n}\right)_{+}, X\right)^{\mathbb{T}} ; \mathbb{F}_{p}\right),
$$

which we call the continuous homology of $X^{h \mathbb{T}}$. Since homology rarely commutes with the formation of limits, the canonical map

$$
H_{*}\left(X^{h \mathbb{T}} ; \mathbb{F}_{p}\right) \rightarrow H_{*}^{c}\left(X^{h \mathbb{T}} ; \mathbb{F}_{p}\right)
$$

is usually not an isomorphism. The Segal conjecture provides striking examples of this phenomenon.

The spectral sequence will be strongly convergent to $H_{*}^{c}\left(X^{h \mathbb{T}} ; \mathbb{F}_{p}\right)$ if the criterion $R E_{* *}^{\infty}=0$ of [Bo99, 7.4] is satisfied, for which it certainly suffices that in each bidegree $(s, t)$ we have $E_{s, t}^{r}=E_{s, t}^{\infty}$ for some finite $r=r(s, t)$. We recall that in this setting, with convergence to the limit, strong convergence means that the kernels $F_{s}=\operatorname{ker}\left(A_{-\infty} \rightarrow A_{s}\right)$ form an exhaustive complete Hausdorff filtration of $A_{-\infty}=H_{*}^{c}\left(X^{h \mathbb{T}} ; \mathbb{F}_{p}\right)$, and that there are isomorphisms $F_{s} / F_{s-1} \cong E_{s}^{\infty}$ for all $s$. When $R E_{* *}^{\infty}=0$, these isomorphisms arise from the upper quadrangle (derived from [Bo99, 5.6 and 5.9]) in the following commuta- 
tive diagram:

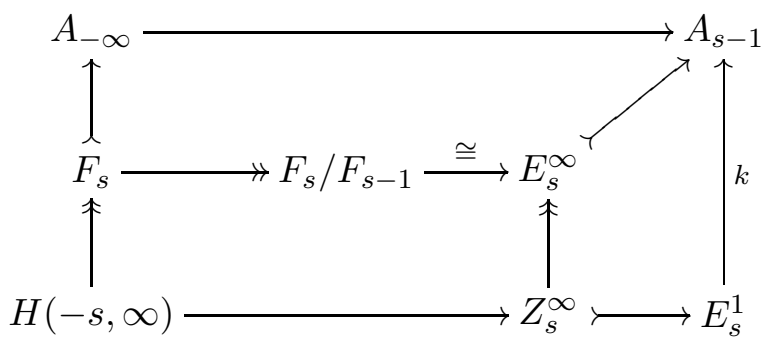

The right hand quadrangle is a pull-back, and the outer rectangle is obtained by applying $F(-, X)^{\mathbb{T}}$ and continuous homology to the commutative square below.

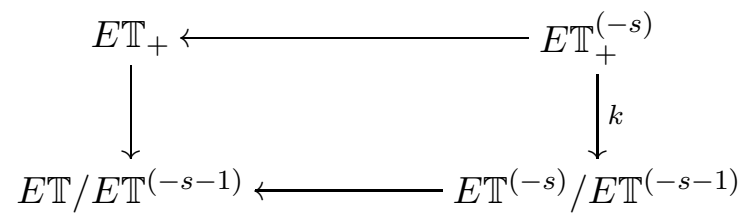

When discussing products, we shall find it more convenient to view the isomorphisms $F_{s} / F_{s-1} \cong E_{s}^{\infty}$ as being determined by the lower and right hand part of (2.4).

We now turn to giving a convenient description of the $E^{2}$-term of the homological homotopy fixed point spectral sequence. By the group cohomology of the circle group $\mathbb{T}$, with coefficients in some discrete group $M$, we mean the singular cohomology of its classifying space $B \mathbb{T}$, or equivalently, the $\mathbb{T}$-equivariant cohomology of the universal space $E \mathbb{T}$ :

$$
H_{g p}^{-*}(\mathbb{T} ; M)=H^{-*}(B \mathbb{T} ; M)=H_{\mathbb{T}}^{-*}(E \mathbb{T} ; M) .
$$

The latter can be computed from the cellular chain complex given by the $\mathbb{T}$ equivariant skeletal filtration of $E \mathbb{T}$, cf. [GM95, Ch. 10]. For $M=H_{t}\left(X ; \mathbb{F}_{p}\right)=$ $\pi_{t}\left(H \mathbb{F}_{p} \wedge X\right)$ we can recognize that cellular chain complex as the row $\left(E_{*, t}^{1}, d^{1}\right)$. This uses the canonical weak equivalence (of e.g. [LMS86, III.1])

$$
\nu: H \mathbb{F}_{p} \wedge F(Z, X)^{\mathbb{T}} \rightarrow F\left(Z, H \mathbb{F}_{p} \wedge X\right)^{\mathbb{T}}
$$

for each finite $\mathbb{T}$-CW spectrum $Z$, applied in the cases $Z=E \mathbb{T}^{(-s)} / E \mathbb{T}^{(-s-1)}$ and $Z=E \mathbb{T}^{(-s+1)} / E \mathbb{T}^{(-s-1)}$ to identify the groups and the differentials, respectively. Therefore the $E^{2}$-term of the homological spectral can be expressed as

$$
E_{s, t}^{2}=H_{g p}^{-s}\left(\mathbb{T} ; H_{t}\left(X ; \mathbb{F}_{p}\right)\right) \cong H_{g p}^{-s}\left(\mathbb{T} ; \mathbb{F}_{p}\right) \otimes H_{t}\left(X ; \mathbb{F}_{p}\right)
$$


Furthermore, $H_{g p}^{*}\left(\mathbb{T} ; \mathbb{F}_{p}\right)=P(y)$ is the polynomial algebra on the Euler class $y \in H_{g p}^{2}\left(\mathbb{T} ; \mathbb{F}_{p}\right)$ of the canonical line bundle over $B \mathbb{T}$. Thus

$$
E_{* *}^{2}=P(y) \otimes H_{*}\left(X ; \mathbb{F}_{p}\right)
$$

with $y$ in bidegree $(-2,0)$ and $H_{t}\left(X ; \mathbb{F}_{p}\right)$ in bidegree $(0, t)$. See GM95, Ch. 14] for a discussion of related spectral sequences.

Proposition 2.1 There is a natural homological spectral sequence of $A_{*}$ comodules

$$
E_{* *}^{2}(X)=H_{g p}^{-*}\left(\mathbb{T} ; H_{*}\left(X ; \mathbb{F}_{p}\right)\right)=P(y) \otimes H_{*}\left(X ; \mathbb{F}_{p}\right)
$$

with $y$ in bidegree $(-2,0)$, converging conditionally to the continuous homology $H_{*}^{c}\left(X^{h \mathbb{T}} ; \mathbb{F}_{p}\right)$. We call this the homological homotopy fixed point spectral sequence. If $H_{*}\left(X ; \mathbb{F}_{p}\right)$ is finite in each degree, or the spectral sequence collapses at a finite stage, then the spectral sequence is strongly convergent.

Remark 2.2 (a) So far, $E^{r}(X)$ was just defined as an additive spectral sequence, but we shall later (in Proposition 4.1) justify the reference to the algebra structure in $P(y)$ by showing that when $X$ is a $\mathbb{T}$-equivariant commutative $S$ algebra, then $E^{r}(X)$ is an algebra spectral sequence. In the special case when $X=S$ the $E^{2}$-term is $P(y)$ and the algebra structure is precisely that of the polynomial algebra $P(y)$. A general (naively) $\mathbb{T}$-equivariant spectrum $X$ can be considered as a (naively) $\mathbb{T}$-equivariant $S$-module [MM02, IV.2.8(iv)], and its homological homotopy fixed point spectral sequence $E^{r}(X)$ becomes a module spectral sequence over the collapsing algebra spectral sequence $E^{r}(S)$ with $E^{2}(S)=E^{\infty}(S)=P(y)$. In this sense the expression for $E^{2}(X)$ describes a natural $P(y)$-module structure on $E^{r}(X)$.

(b) As noted in the introduction, it is rather more traditional to apply the homotopy group functor $\pi_{*}(-)$ to the tower of fibrations (2.2), to obtain an unrolled exact couple and a conditionally convergent (homotopical) homotopy fixed point spectral sequence

$$
E_{s, t}^{2}=H_{g p}^{-s}\left(\mathbb{T} ; \pi_{t}(X)\right) \Longrightarrow \pi_{s+t}\left(X^{h \mathbb{T}}\right) .
$$

However, this is not the spectral sequence that we are considering. Earlier work by Ch. Ausoni and the second author [AuR02, Ch. 4], as well as recent work by S. Lunøe-Nielsen (and the second author) [L-N], supports the assertion that the homological spectral sequence is an interesting object.

(c) In view of the natural weak equivalence (2.5), applied to the various skeletal filtration quotients $Z=E \mathbb{T}^{(m)} / E \mathbb{T}^{(n)}$, the homological homotopy fixed point 
spectral sequence for the $\mathbb{T}$-equivariant spectrum $X$ is in fact isomorphic to the homotopical homotopy fixed point spectral sequence for $H \mathbb{F}_{p} \wedge X$, where $\mathbb{T}$ acts trivially on the Eilenberg-Mac Lane spectrum. More precisely, they have isomorphic defining Cartan-Eilenberg systems, in the sense of [CE56, XV.7].

(d) In general there can be arbitrarily long differentials in the homological homotopy fixed point spectral sequence. For example, when $X=S\left(\mathbb{C}^{r}\right)_{+}$with the free $\mathbb{T}$-action, the differentials $d_{s, 0}^{2 r}$ are nonzero for all even $s=-2 n \leq 0$. The point of Theorem 1.2 is that this rarely happens when $X=R$ is a $\mathbb{T}$ equivariant commutative $S$-algebra.

\section{Differentials}

We now make the differentials in the homological homotopy fixed point spectral sequence more explicit, as obstructions to extending equivariant maps.

Consider a class $x \in H_{t}\left(X ; \mathbb{F}_{p}\right)$, represented at the $E^{2}$-term of the homological spectral sequence in bidegree $(0, t)$. We briefly write $H=H \mathbb{F}_{p}$ for the $\bmod p$ Eilenberg-Mac Lane spectrum. Then $x$ can be represented as a non-equivariant map $S^{t} \rightarrow H \wedge X$, or equivalently as a $\mathbb{T}$-equivariant map

$$
x: S(\mathbb{C})_{+} \wedge S^{t} \rightarrow H \wedge X .
$$

Here $\mathbb{T}$ acts on $S(\mathbb{C})_{+}$(freely off the base point) and $X$, but not on $S^{t}$ or $H$. The condition that $x \in E_{0, t}^{2}=H_{t}\left(X ; \mathbb{F}_{p}\right)$ survives to the $E^{2 r}$-term, i.e., that all differentials $d^{2}(x), \ldots, d^{2 r-2}(x)$ vanish, is equivalent to $x$ being in the image from $H_{t}\left(F\left(S\left(\mathbb{C}^{r}\right)_{+}, X\right)^{\mathbb{T}} ; \mathbb{F}_{p}\right)$ under the map induced by restriction along $S(\mathbb{C})_{+} \subset S\left(\mathbb{C}^{r}\right)_{+}$. This is in turn equivalent to the existence of a $\mathbb{T}$-equivariant extension

$$
x^{\prime}: S\left(\mathbb{C}^{r}\right)_{+} \wedge S^{t} \rightarrow H \wedge X
$$

of $x$ along $S(\mathbb{C})_{+} \subset S\left(\mathbb{C}^{r}\right)_{+}$, in view of the natural naively equivariant weak equivalence

$$
\nu: H \wedge F\left(S\left(\mathbb{C}^{r}\right)_{+}, X\right) \rightarrow F\left(S\left(\mathbb{C}^{r}\right)_{+}, H \wedge X\right) .
$$

(To establish this equivalence, use that $S\left(\mathbb{C}^{r}\right)_{+}$is a finite $\mathbb{T}-\mathrm{CW}$ complex. We are considering maps from free $\mathbb{T}$-CW complexes into these spectra, so only the naive notion of a $\mathbb{T}$-equivariant equivalence is required.)

Suppose that $x \in E_{0, t}^{2 r}$ has survived to the $E^{2 r}$-term, so that such a $\mathbb{T}$ equivariant extension $x^{\prime}$ exists. Then by the construction of the homological spectral sequence, the differential

$$
d^{2 r}(x) \in E_{-2 r, t+2 r-1}^{2 r}
$$


is the obstruction to extending $x^{\prime}$ further along $S\left(\mathbb{C}^{r}\right)_{+} \subset S\left(\mathbb{C}^{r+1}\right)_{+}$to an equivariant map

$$
x^{\prime \prime}: S\left(\mathbb{C}^{r+1}\right)_{+} \wedge S^{t} \rightarrow H \wedge X .
$$

We put the obvious right adjoints of these maps together in a diagram.

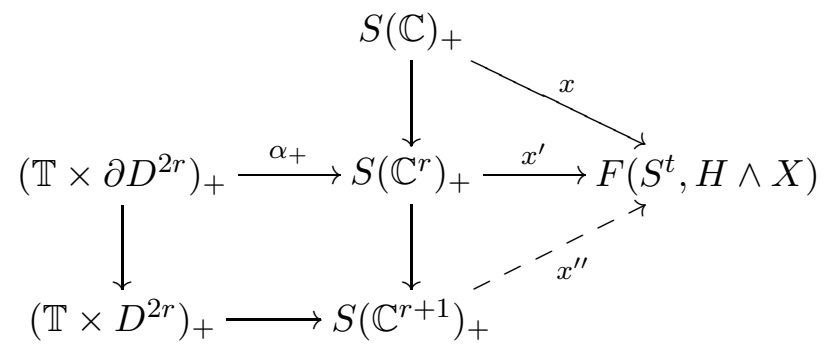

Recalling from (2.1) that $S\left(\mathbb{C}^{r+1}\right)_{+}$is obtained from $S\left(\mathbb{C}^{r}\right)_{+}$by adjoining a free $\mathbb{T}$-cell along the action map $\alpha: \mathbb{T} \times \partial D^{2 r} \rightarrow S\left(\mathbb{C}^{r}\right)$, the obstruction to such an extension is precisely the obstruction to extending the equivariant map $x^{\prime} \circ \alpha_{+}$ from $\left(\mathbb{T} \times \partial D^{2 r}\right)_{+}$over $\left(\mathbb{T} \times D^{2 r}\right)_{+}$. By adjunction, this equals the obstruction to extending the underlying non-equivariant map $x^{\prime}: \partial D_{+}^{2 r}=S\left(\mathbb{C}^{r}\right)_{+} \rightarrow F\left(S^{t}, H \wedge\right.$ $X)$ over $D_{+}^{2 r}$. In terms of the preferred stable splitting

$$
\partial D_{+}^{2 r} \simeq S^{2 r-1} \vee D_{+}^{2 r}
$$

(induced by the pinch map from $\partial D_{+}^{2 r}$ to $S^{2 r-1}$ and the inclusion to $D_{+}^{2 r}$ ) this equals the restriction of the non-equivariant map $x^{\prime}$ to the stable summand $S^{2 r-1}$. Its left adjoint again is a map

$$
\delta x: S^{2 r-1} \wedge S^{t} \rightarrow H \wedge X,
$$

which represents $d^{2 r}(x)$. We summarize:

Lemma 3.1 Let $x \in E_{0, t}^{2 r} \subset H_{t}\left(X ; \mathbb{F}_{p}\right)$ be represented by a $\mathbb{T}$-equivariant map $x: S(\mathbb{C})_{+} \wedge S^{t} \rightarrow H \wedge X$ that extends to a $\mathbb{T}$-equivariant map $x^{\prime}: S\left(\mathbb{C}^{r}\right)_{+} \wedge S^{t} \rightarrow$ $H \wedge X$. Then $d^{2 r}(x)=y^{r} \cdot \delta x$, where $\delta x \in H_{t+2 r-1}\left(X ; \mathbb{F}_{p}\right)$ is represented by $x^{\prime}$ considered as a non-equivariant map, restricted to the stable summand $S^{2 r-1} \wedge S^{t}$ of $S\left(\mathbb{C}^{r}\right)_{+} \wedge S^{t}$.

The extended map $x^{\prime}$ represents a class in the homology of $F\left(S\left(\mathbb{C}^{r}\right)_{+}, X\right)^{\mathbb{T}}$, and considering $x^{\prime}$ as a non-equivariant map amounts to following the map

$$
\varphi: F\left(S\left(\mathbb{C}^{r}\right)_{+}, X\right)^{\mathbb{T}} \rightarrow F\left(S_{+}^{2 r-1}, X\right)
$$

that forgets the $\mathbb{T}$-equivariance. There is a canonical map

$$
\nu: X \wedge D S_{+}^{2 r-1} \rightarrow F\left(S_{+}^{2 r-1}, X\right)
$$


where $D S_{+}^{2 r-1}=F\left(S_{+}^{2 r-1}, S\right)$ is the functional dual of $S_{+}^{2 r-1}$, which is a weak equivalence since $S_{+}^{2 r-1}$ is a finite $\mathrm{CW}$ complex. Hence there is a natural isomorphism

$$
\nu: H_{*}\left(X ; \mathbb{F}_{p}\right) \otimes H^{-*}\left(S^{2 r-1} ; \mathbb{F}_{p}\right) \stackrel{\cong}{\rightrightarrows} H_{*}\left(F\left(S_{+}^{2 r-1}, X\right) ; \mathbb{F}_{p}\right),
$$

where we have identified the spectrum homology $H_{*}\left(D S_{+}^{2 r-1} ; \mathbb{F}_{p}\right)$ with the space-level cohomology $H^{-*}\left(S^{2 r-1} ; \mathbb{F}_{p}\right)$. We write $H^{*}\left(S^{2 r-1} ; \mathbb{F}_{p}\right)=E\left(\iota_{2 r-1}\right)$, where $\iota_{2 r-1}$ is the canonical generator in degree $(2 r-1)$ and $E(-)$ denotes the exterior algebra.

Proposition 3.2 The composite map

$$
\begin{aligned}
& H_{*}\left(F\left(S\left(\mathbb{C}^{r}\right)_{+}, X\right)^{\mathbb{T}} ; \mathbb{F}_{p}\right) \stackrel{\varphi_{*}}{\longrightarrow} H_{*}\left(F\left(S_{+}^{2 r-1}, X\right) ; \mathbb{F}_{p}\right) \\
& \stackrel{\nu_{*}}{\cong} H_{*}\left(X ; \mathbb{F}_{p}\right) \otimes H^{-*}\left(S^{2 r-1} ; \mathbb{F}_{p}\right)
\end{aligned}
$$

takes any class $x^{\prime}$ that maps to $x \in E_{0, t}^{2 r} \subset H_{t}\left(X ; \mathbb{F}_{p}\right)$ by the restriction map

$$
H_{*}\left(F\left(S\left(\mathbb{C}^{r}\right)_{+}, X\right)^{\mathbb{T}} ; \mathbb{F}_{p}\right) \rightarrow H_{*}\left(F\left(S(\mathbb{C})_{+}, X\right)^{\mathbb{T}} ; \mathbb{F}_{p}\right)=H_{*}\left(X ; \mathbb{F}_{p}\right)
$$

to the sum

$$
\left(\nu_{*}^{-1} \varphi_{*}\right)\left(x^{\prime}\right)=x \otimes 1+\delta x \otimes \iota_{2 r-1},
$$

where $d^{2 r}(x)=y^{r} \cdot \delta x$ in $E_{-2 r, t+2 r-1}^{2 r}$. Suppressing the power of $y$ we may somewhat imprecisely write this formula as

$$
\varphi_{*}(x)=x \otimes 1+d^{2 r}(x) \otimes \iota_{2 r-1} .
$$

The case $r=1$ of this result says that $d^{2}(x)=y \cdot \sigma x$, and follows e.g. from Ro98, 3.3].

Proof This is really a corollary to Lemma 3.1, but for the observation that the restriction of the non-equivariant $x^{\prime}$ to the subspace $S^{t} \subset S\left(\mathbb{C}^{r}\right)_{+} \wedge S^{t}$ equals the restriction of the non-equivariant $x$ to the same subspace $S^{t} \subset$ $S(\mathbb{C})_{+} \wedge S^{t}$, which in turn corresponds to $x \in E_{0, t}^{2 r}$ under the identification $H_{*}\left(F\left(S(\mathbb{C})_{+}, X\right)^{\mathbb{T}} ; \mathbb{F}_{p}\right)=H_{*}\left(X ; \mathbb{F}_{p}\right)$. There are no signs in these formulas, because the canonical map $\nu$ is derived from the non-symmetric part of the closed monoidal structure on the stable homotopy category. (See e.g. LMS86 III.1].)

Remark 3.3 Lemma 3.1 says that the differential in the homotopy fixed point spectral sequence is essentially the $\mathbb{T}$-equivariant root invariant for $H \wedge X$. A corresponding description of the Mahowald $C_{2}$-equivariant root invariant for $S$ 
can be found in BG95, 2.5]: Let $S^{n+k \alpha}$ denote the $C_{2}$-equivariant sphere that is the one point compactification of $\mathbb{R}^{n} \oplus \mathbb{R}^{k}(-1)$, where $C_{2}$ acts trivially on $\mathbb{R}^{n}$ and by negation on $\mathbb{R}^{k}(-1)$. Given a non-equivariant (stable) map $x: S^{n} \rightarrow S^{0}$, let $x^{\prime}: S^{n+k \alpha} \rightarrow S^{0}$ be a $C_{2}$-equivariant extension of $x$ with $k$ maximal. Then the $C_{2}$-equivariant root invariant of $x$ contains the nonequivariant map $x^{\prime}: S^{n+k} \rightarrow S^{0}$ underlying $x^{\prime}$.

\section{Commutative $S$-algebras}

Now suppose that $X=R$ is a naively $\mathbb{T}$-equivariant commutative $S$-algebra, i.e., a commutative $S$-algebra with a continuous point-set level action by the circle group $\mathbb{T}$, through commutative $S$-algebra maps. We shall be concerned with the homotopy fixed points of $R$, rather than its genuine fixed points, so only this weak notion of an equivariant spectrum will be needed. Cf. GM95, Ch. 1]. Our principal example is $R=T H H(B)$, the topological Hochschild homology spectrum of a non-equivariant commutative $S$-algebra $B$. The cyclic structure on topological Hochschild homology [EKMM97, Ch. IX] then provides the relevant $\mathbb{T}$-action. In terms of the tensored structure on commutative $S$-algebras over topological spaces, one model for the topological Hochschild homology spectrum is $\operatorname{THH}(B)=B \otimes \mathbb{T}$, and then $\mathbb{T}$ acts naturally through commutative $S$-algebra maps by group multiplication in the $\mathbb{T}$-factor.

In this situation the homotopy fixed point spectrum $R^{h \mathbb{T}}=F\left(E \mathbb{T}_{+}, R\right)^{\mathbb{T}}$ is also a commutative $S$-algebra. Writing $\mu: R \wedge R \rightarrow R$ for the $\mathbb{T}$-equivariant multiplication map of $R$, the corresponding multiplication map for $R^{h \mathbb{T}}$ is given by the composite

$$
\begin{aligned}
F\left(E \mathbb{T}_{+}, R\right)^{\mathbb{T}} \wedge F\left(E \mathbb{T}_{+}, R\right)^{\mathbb{T}} & \stackrel{\wedge}{\rightarrow} F\left((E \mathbb{T} \times E \mathbb{T})_{+}, R \wedge R\right)^{\mathbb{T}} \\
& \stackrel{\mu_{\#} \Delta_{+}^{\#}}{\longrightarrow} F\left(E \mathbb{T}_{+}, R\right)^{\mathbb{T}} .
\end{aligned}
$$

Here $\wedge$ smashes together two $\mathbb{T}$-equivariant maps $\Sigma^{\infty} E \mathbb{T}_{+} \rightarrow R$, and considers the resulting $(\mathbb{T} \times \mathbb{T})$-equivariant map as being $\mathbb{T}$-equivariant via the diagonal action. The map $\mu_{\#}$ composes on the left by $\mu: R \wedge R \rightarrow R$, while the map $\Delta_{+}^{\#}$ composes on the right by the diagonal map $\Delta_{+}: E \mathbb{T}_{+} \rightarrow(E \mathbb{T} \times E \mathbb{T})_{+}$. Since $\mu$ is commutative and $\Delta_{+}$is cocommutative, the resulting multiplication on $R^{h \mathbb{T}}$ is also strictly commutative.

Writing $\eta: S \rightarrow R$ for the $\mathbb{T}$-equivariant unit map of $R$, the corresponding unit map for $R^{h \mathbb{T}}$ is the composite

$$
S \rightarrow F\left(E \mathbb{T}_{+}, S\right)^{\mathbb{T}} \stackrel{\eta_{\#}}{\longrightarrow} F\left(E \mathbb{T}_{+}, R\right)^{\mathbb{T}}
$$


To define the first map, we must use that $\mathbb{T}$ acts trivially on $S$.

The same constructions can be applied for the $\mathbb{T}$-CW skeleta of $E \mathbb{T}$, so each $F\left(S\left(\mathbb{C}^{n}\right)_{+}, R\right)^{\mathbb{T}}$ is also a commutative $S$-algebra. In particular, the continuous homology $H_{*}^{c}\left(R^{h \mathbb{T}} ; \mathbb{F}_{p}\right)$ is a limit of graded commutative algebras, and is therefore itself a graded commutative algebra.

Proposition 4.1 Let $R$ be a $\mathbb{T}$-equivariant commutative $S$-algebra. Then the homological homotopy fixed point spectral sequence

$$
E_{* *}^{2}(R)=P(y) \otimes H_{*}\left(R ; \mathbb{F}_{p}\right) \Longrightarrow H_{*}^{c}\left(R^{h \mathbb{T}} ; \mathbb{F}_{p}\right)
$$

is an $A_{*}$-comodule algebra spectral sequence, where $P(y)$ is the polynomial algebra on $y$ in bidegree $(-2,0)$, and $H_{*}\left(R ; \mathbb{F}_{p}\right)$ has the Pontryagin product.

Proof The algebra product in $E^{r}(R)$ is derived from the $\mathbb{T}$-equivariant commutative $S$-algebra product in $R$ and a $\mathbb{T}$-equivariant cellular approximation $d$ to the diagonal map $\Delta: E \mathbb{T} \rightarrow E \mathbb{T} \times E \mathbb{T}$. The deduction is in principle standard, but due to our homological indexing and perhaps unusual choice of exact couple defining the spectral sequence, it is not so easy to find an applicable reference. (The closest may be a combination of Remark [2.2(c) and [HM03, 4.3.5], adapted from the $G$-Tate construction for a finite group $G$ to the $\mathbb{T}$-homotopy fixed point spectrum.) We therefore provide the following outline.

The product $(\mathbb{T} \times \mathbb{T})$-CW structure on $E \mathbb{T} \times E \mathbb{T}$ can be refined to a $\mathbb{T}$-CW structure, by starting with a $\mathbb{T}$-CW structure on $\mathbb{T} \times \mathbb{T}$ with the diagonal $\mathbb{T}$ action. Fix a choice of a $\mathbb{T}$-equivariant cellular map $d: E \mathbb{T} \rightarrow E \mathbb{T} \times E \mathbb{T}$ that is equivariantly homotopic to $\Delta$.

To shorten the notation, we shall write

$$
E \mathbb{T}_{a}^{m}=E \mathbb{T}^{(m)} / E \mathbb{T}^{(a-1)}
$$

within this proof. Then for all integers $s, s^{\prime}$ and $r \geq 1, d$ induces a map of subquotients

$$
d: E \mathbb{T}_{-s-s^{\prime}}^{-s-s^{\prime}+r-1} \rightarrow E \mathbb{T}_{-s}^{-s+r-1} \wedge E \mathbb{T}_{-s^{\prime}}^{-s^{\prime}+r-1},
$$

since each $\mathbb{T}$ - $m$-cell in the source maps into the $(\mathbb{T} \times \mathbb{T})$ - $m$-skeleton in the product structure on the target, which is the union of products of $\mathbb{T}$ - $k$-cells and $\mathbb{T}$ - $k^{\prime}$-cells with $k \geq-s, k^{\prime} \geq-s^{\prime}$ and $k+k^{\prime} \leq m$. For $s=-2 n$ and $s^{\prime}=-2 n^{\prime}$ non-positive and even, and $r=1$, this is a map

$$
d: \mathbb{T}_{+} \wedge S^{2 n+2 n^{\prime}} \rightarrow \mathbb{T}_{+} \wedge S^{2 n} \wedge \mathbb{T}_{+} \wedge S^{2 n^{\prime}}
$$

homotopic to the $\left(2 n+2 n^{\prime}\right)$-th suspension of the diagonal $\mathbb{T}_{+} \rightarrow(\mathbb{T} \times \mathbb{T})_{+}$. 
For integers $a \leq b$, let

$$
H(a, b)=H_{*}\left(F\left(E \mathbb{T}_{a}^{b-1}, R\right)^{\mathbb{T}} ; \mathbb{F}_{p}\right)
$$

be the finite terms of a Cartan-Eilenberg system [CE56, XV.7], with $E_{s}^{1}=$ $H(-s,-s+1)$. Applying $F(-, R)^{\mathbb{T}}$ and homology to $d$, we obtain homomorphisms

$$
\varphi_{r}: H(-s,-s+r) \otimes H\left(-s^{\prime},-s^{\prime}+r\right) \rightarrow H\left(-s-s^{\prime},-s-s^{\prime}+r\right)
$$

for all integers $s, s^{\prime}$ and $r \geq 1$. For $s=-2 n, s^{\prime}=-2 n^{\prime}$ non-positive and $r=1$, this is the homomorphism

$$
\varphi_{1}: E_{-2 n}^{1} \otimes E_{-2 n^{\prime}}^{1} \rightarrow E_{-2 n-2 n^{\prime}}^{1}
$$

that takes $y^{n} \cdot x \otimes y^{n^{\prime}} \cdot x^{\prime}$ to $y^{n+n^{\prime}} \cdot \mu_{*}\left(x \otimes x^{\prime}\right)$, under the identification $E_{-2 n, t}^{1}=$ $y^{n} \cdot H_{t}\left(R ; \mathbb{F}_{p}\right)$ from Section 2. So $E^{1}=E^{2}$ equipped with the product $\varphi_{1}$ is isomorphic to the tensor product of the polynomial algebra $P(y)$ and $H_{*}\left(R ; \mathbb{F}_{p}\right)$ with the Pontryagin product $\mu_{*}$.

To verify that the spectral sequence differentials $d^{r}$ are derivations, so that each term $E^{r+1}$ inductively inherits an algebra structure from $E^{r}$, it suffices to check that we have a multiplicative Cartan-Eilenberg system, i.e., that the pairings $\varphi_{r}$ satisfy the relation

$$
\delta\left(\varphi_{r}\left(z \otimes z^{\prime}\right)\right)=\varphi_{1}\left(\delta(z) \otimes \eta\left(z^{\prime}\right)\right)+(-1)^{|z|} \varphi_{1}\left(\eta(z) \otimes \delta\left(z^{\prime}\right)\right)
$$

in $E_{s+s^{\prime}-r}^{1}$, for $z \in H(-s,-s+r)$ of degree $|z|$ and $z^{\prime} \in H\left(-s^{\prime},-s^{\prime}+r\right)$. Here $\delta: H(-s,-s+r) \rightarrow H(-s+r,-s+r+1)=E_{s-r}^{1}$ is the degree $(-1)$ homomorphism induced by the stable connecting map

$$
\delta: \Sigma^{-1} E \mathbb{T}_{-s+r}^{-s+r} \rightarrow E \mathbb{T}_{-s}^{-s+r-1}
$$

of the obvious triple, and $\eta: H(-s,-s+r) \rightarrow H(-s,-s+1)=E_{s}^{1}$ is the homomorphism induced by the inclusion

$$
\eta: E \mathbb{T}_{-s}^{-s} \rightarrow E \mathbb{T}_{-s}^{-s+r-1} .
$$

We use similar notations with $s^{\prime}$ and $s+s^{\prime}$ in place of $s$. The sufficiency of this condition can be read directly off from the definition of the differentials in the spectral sequence associated to an unrolled exact couple, or obtained from Ma54 or [Mc01, Ex. 2.2.1].

In geometric terms, (4.2) asks that the composite map

$$
\Sigma^{-1} E \mathbb{T}_{-s-s^{\prime}+r}^{-s-s^{\prime}+r} \stackrel{\delta}{\rightarrow} E \mathbb{T}_{-s-s^{\prime}}^{-s-s^{\prime}+r-1} \stackrel{d}{\rightarrow} E \mathbb{T}_{-s}^{-s+r-1} \wedge E \mathbb{T}_{-s^{\prime}}^{-s^{\prime}+r-1}
$$


is homotopic, as a map of $\mathbb{T}$-equivariant spectra, to the sum of the two composite maps

$$
\Sigma^{-1} E \mathbb{T}_{-s-s^{\prime}+r}^{-s-s^{\prime}+r} \stackrel{d}{\rightarrow} \Sigma^{-1} E \mathbb{T}_{-s+r}^{-s+r} \wedge E \mathbb{T}_{-s^{\prime}}^{-s^{\prime}} \stackrel{\delta \wedge \eta}{\longrightarrow} E \mathbb{T}_{-s}^{-s+r-1} \wedge E \mathbb{T}_{-s^{\prime}}^{-s^{\prime}+r-1}
$$

and

$$
\Sigma^{-1} E \mathbb{T}_{-s-s^{\prime}+r}^{-s-s^{\prime}+r} \stackrel{(-1)^{s} d}{\longrightarrow} E \mathbb{T}_{-s}^{-s} \wedge \Sigma^{-1} E \mathbb{T}_{-s^{\prime}+r}^{-s^{\prime}+r} \stackrel{\eta \wedge \delta}{\longrightarrow} E \mathbb{T}_{-s}^{-s+r-1} \wedge E \mathbb{T}_{-s^{\prime}}^{-s^{\prime}+r-1} .
$$

There is only something to check when $s, s^{\prime}$ and $r$ are all even, with $s$ and $s^{\prime}$ non-positive. The common source of the $\mathbb{T}$-equivariant stable maps to be compared is then $\Sigma^{-1} \mathbb{T}_{+} \wedge S^{-s-s^{\prime}+r}$, so by an adjunction we may as well compare non-equivariant maps from $S^{-s-s^{\prime}+r-1}$ to

$$
E \mathbb{T}_{-s}^{-s+r-1} \wedge E \mathbb{T}_{-s^{\prime}}^{-s^{\prime}+r-1} \simeq\left(S^{-s+r-1} \vee S^{-s}\right) \wedge\left(S^{-s^{\prime}+r-1} \vee S^{-s^{\prime}}\right)
$$

The projection to the stable summand $\Sigma E \mathbb{T}^{(-s-1)} \wedge \Sigma E \mathbb{T}^{\left(-s^{\prime}-1\right)}=S^{-s} \wedge S^{-s^{\prime}}$ in the target is trivial for each of the three maps, since in each case two subsequent maps in a cofiber sequence occur as a factor of the composite map. The projections to the summands $E \mathbb{T}^{(-s+r-1)} \wedge \Sigma E \mathbb{T}^{\left(-s^{\prime}-1\right)}=S^{-s+r-1} \wedge S^{-s^{\prime}}$ and $\Sigma E \mathbb{T}^{(-s-1)} \wedge E \mathbb{T}^{\left(-s^{\prime}+r-1\right)}=S^{-s} \wedge S^{-s^{\prime}+r-1}$ agree as required, by the same kind of homotopy as in (4.1).

Finally, in the presence of strong convergence due to the vanishing of Boardman's obstruction group $R E_{* *}^{\infty}$, we claim that the isomorphisms $F_{s} / F_{s-1} \cong$ $E_{s}^{\infty}$ take the associated graded algebra structure derived from the product on $H_{*}^{c}\left(R^{h \mathbb{T}} ; \mathbb{F}_{p}\right)$ to the algebra structure on $E^{\infty}$-term. These isomorphisms are obtained by descent to subquotients, as in (2.4), from the homomorphisms

$$
\lim _{m} H_{*}\left(F\left(E \mathbb{T}_{-s}^{m}, R\right)^{\mathbb{T}} ; \mathbb{F}_{p}\right)=H(-s, \infty) \rightarrow H(-s,-s+1)=E_{s}^{1},
$$

so it suffices to verify that the following diagram commutes:

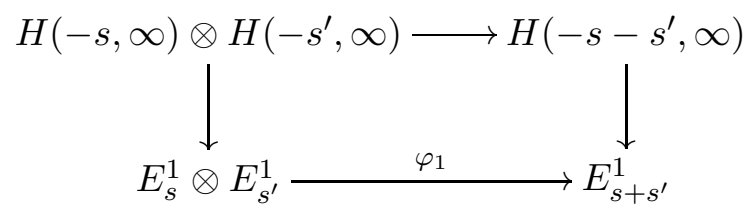

This follows immediately from the commutativity of the following diagram, where $d$ is the diagonal approximation and the vertical maps are inclusions:

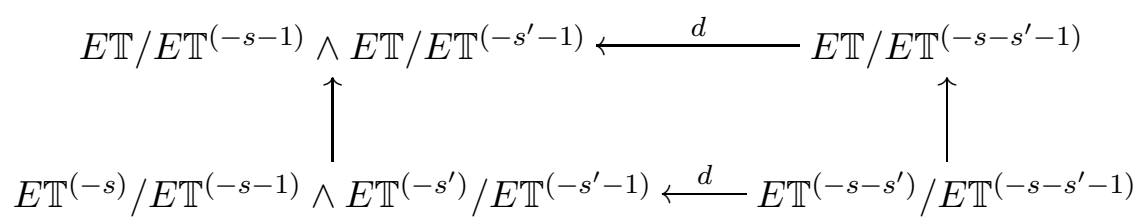


Commutative $S$-algebras are $E_{\infty}$ ring spectra ([EKMM97, II.4]), and are in particular also $H_{\infty}$ ring spectra ([EKMM97, III.5] and [LMS86, VII.2]). Hence there are Dyer-Lashof operations $Q^{i}$ acting on their mod $p$ homology algebras BMMS86, III.1]. Recall that $Q^{i}$ is a natural transformation

$$
Q^{i}: H_{t}\left(R ; \mathbb{F}_{p}\right) \rightarrow H_{t+i q}\left(R ; \mathbb{F}_{p}\right)
$$

for all integers $t$, where $q=2 p-2$. We also include their composites $\beta Q^{i}$ with the homology Bockstein operation $\beta: H_{t}\left(R ; \mathbb{F}_{p}\right) \rightarrow H_{t-1}\left(R ; \mathbb{F}_{p}\right)$ as generators of the Dyer-Lashof algebra. For $p=2$ the standard notation is to write $Q^{2 i}$ and $Q^{2 i-1}$ for the operations that would otherwise be called $Q^{i}$ and $\beta Q^{i}$, respectively.

As noted before Proposition 4.1, the commutative $S$-algebra structure on $R^{h \mathbb{T}}=$ $F\left(E \mathbb{T}_{+}, R\right)^{\mathbb{T}}$ restricts to one on each $F\left(S\left(\mathbb{C}^{n}\right)_{+}, R\right)^{\mathbb{T}}$, so each algebra homomorphism

$$
H_{*}^{c}\left(R^{h \mathbb{T}} ; \mathbb{F}_{p}\right) \rightarrow H_{*}\left(F\left(S\left(\mathbb{C}^{n}\right)_{+}, R\right)^{\mathbb{T}} ; \mathbb{F}_{p}\right)
$$

commutes with the Dyer-Lashof operations in the source and in the target. In particular, for $n=1$ the identification $F\left(S(\mathbb{C})_{+}, R\right)^{\mathbb{T}} \cong R$ lets us recognize the Dyer-Lashof operations in the target as those in $H_{*}\left(R ; \mathbb{F}_{p}\right)$. This action by Dyer-Lashof operations on the vertical axis $E_{0, *}^{2}$ can be algebraically extended to an action on the full $E^{2}$-term, by the formula $\beta^{\epsilon} Q^{i}\left(y^{n} \cdot x\right)=y^{n} \cdot \beta^{\epsilon} Q^{i}(x)$. Our next result shows that this action extends further to all terms of the homological homotopy fixed point spectral sequence.

Proposition $4.2 \quad R$ be a $\mathbb{T}$-equivariant commutative $S$-algebra and let $E^{r}(R)$ be its homological homotopy fixed point spectral sequence. Then for each element $x \in E_{0, t}^{2 r} \subset H_{t}\left(R ; \mathbb{F}_{p}\right)$ we have the relation

$$
d^{2 r}\left(\beta^{\epsilon} Q^{i}(x)\right)=\beta^{\epsilon} Q^{i}\left(d^{2 r}(x)\right),
$$

for every integer $i$ and $\epsilon \in\{0,1\}$. Here the right hand side should be interpreted as follows: If $d^{2 r}(x)=y^{r} \cdot \delta x$ with $\delta x \in H_{t+2 r-1}\left(R ; \mathbb{F}_{p}\right)$ then $\beta^{\epsilon} Q^{i}\left(d^{2 r}(x)\right)=$ $y^{r} \cdot \beta^{\epsilon} Q^{i}(\delta x)$.

The case $r=1$ appears as $[\mathrm{AnR}, 5.9$ ], with a proof that we generalize as follows.

Proof Let $x \in H_{t}\left(R ; \mathbb{F}_{p}\right)$ and suppose that $x$ survives to the $E^{2 r}$-term. Then there exists an extension $x^{\prime} \in H_{t}\left(F\left(S\left(\mathbb{C}^{r}\right)_{+}, R\right)^{\mathbb{T}} ; \mathbb{F}_{p}\right)$ of $x$ over the restriction map

$$
F\left(S\left(\mathbb{C}^{r}\right)_{+}, R\right)^{\mathbb{T}} \rightarrow F\left(S(\mathbb{C})_{+}, R\right)^{\mathbb{T}} \cong R
$$


of commutative $S$-algebras, and $z^{\prime}=\beta^{\epsilon} Q^{i}\left(x^{\prime}\right)$ is an extension of $z=\beta^{\epsilon} Q^{i}(x)$ over the same map, by naturality of the Dyer-Lashof operations. The maps $\varphi$ and $\nu$ from Proposition 3.2 are both maps of commutative $S$-algebras, and therefore induce algebra homomorphisms $\varphi_{*}$ and $\nu_{*}$ that commute with the Dyer-Lashof operations. Thus

$$
\left(\nu_{*}^{-1} \varphi_{*}\right)\left(\beta^{\epsilon} Q^{i}\left(x^{\prime}\right)\right)=\beta^{\epsilon} Q^{i}(x) \otimes 1+\delta z \otimes \iota_{2 r-1}
$$

where $d^{2 r}\left(\beta^{\epsilon} Q^{i}(x)\right)=y^{r} \cdot \delta z$, is equal to

$$
\beta^{\epsilon} Q^{i}\left(\left(\nu_{*}^{-1} \varphi_{*}\right)\left(x^{\prime}\right)\right)=\beta^{\epsilon} Q^{i}\left(x \otimes 1+\delta x \otimes \iota_{2 r-1}\right)
$$

where $d^{2 r}(x)=y^{r} \cdot \delta x$. Now the Dyer-Lashof operations on the homology of the smash product $R \wedge D S_{+}^{2 r-1}$ are given by a Cartan formula, and on the tensor factor $H_{*}\left(D S_{+}^{2 r-1} ; \mathbb{F}_{p}\right) \cong H^{-*}\left(S^{2 r-1} ; \mathbb{F}_{p}\right)$ the operation $\beta^{\epsilon} Q^{i}$ corresponds to the Steenrod operation $\beta^{\epsilon} P^{-i}$, by BMMS86, III.1.2]. But the latter operations all act trivially on $H^{*}\left(S^{2 r-1} ; \mathbb{F}_{p}\right)$, except for $P^{0}=1$, so the Cartan formula gives

$$
\beta^{\epsilon} Q^{i}\left(x \otimes 1+\delta x \otimes \iota_{2 r-1}\right)=\beta^{\epsilon} Q^{i}(x) \otimes 1+\beta^{\epsilon} Q^{i}(\delta x) \otimes \iota_{2 r-1} .
$$

Identifying this with (4.3) and comparing the coefficients of $\iota_{2 r-1}$ we obtain the identity

$$
\delta z=\beta^{\epsilon} Q^{i}(\delta x)
$$

as claimed.

The homological homotopy fixed point spectral sequence for $S$ itself is particularly simple. For $H_{*}\left(S ; \mathbb{F}_{p}\right)=\mathbb{F}_{p}$, so the spectral sequence collapses to

$$
E_{* *}^{2}(S)=P(y),
$$

concentrated on the horizontal axis. Hence each power of $y$ is an infinite cycle, i.e., $d^{2 r}\left(y^{n}\right)=0$ for all $r \geq 1$ and $n$. The spectral sequence $E^{*}(X)$ for a general $\mathbb{T}$-equivariant $S$-module $X$ is a module over the one for $S$, so the Leibniz formula for the module pairing immediately yields part (a) of the following result.

Lemma 4.3 Let $X$ be any $\mathbb{T}$-equivariant $S$-module.

(a) The differentials in the homological homotopy fixed point spectral sequence converging to $H_{*}^{c}\left(X^{h \mathbb{T}} ; \mathbb{F}_{p}\right)$ satisfy the relation

$$
d^{2 r}\left(y^{n} \cdot x\right)=y^{n} \cdot d^{2 r}(x)
$$

for all $x \in E_{0, *}^{2 r} \subset H_{*}\left(X ; \mathbb{F}_{p}\right), 2 r \geq 2$ and $n \geq 0$. 
(b) Each class in $E_{-2 n, t}^{2 r}$ has the form $y^{n} \cdot x$ for a class $x \in E_{0, t}^{2 r} \subset H_{t}\left(X ; \mathbb{F}_{p}\right)$. Hence the spectral sequence is completely determined by the differentials that originate on the vertical axis.

(c) The $y$-torsion in $E_{* *}^{2 r}$ has height strictly less than $r$, and is concentrated in filtrations $-2 r+4 \leq s \leq 0$.

Proof It remains to prove parts (b) and (c), which we do by induction on $r$. The claims for $r=1$ are clear from Propositions 2.1 and 4.1.

If $z=y^{n} \cdot x \in E_{-2 n, t}^{2 r}$ survives to the $E^{2 r+2}$-term, then $0=d^{2 r}\left(y^{n} \cdot x\right)=$ $y^{n} \cdot d^{2 r}(x)$ by part (a). By induction there is no $y$-torsion in or below the filtration of $d^{2 r}(x)$, so $d^{2 r}(x)=0, x$ survives to the $E^{2 r+2}$-term, and we still have $z=y^{n} \cdot x$.

If now $y \cdot z=0$ in the $E^{2 r+2}$-term, then $y \cdot z$ in the $E^{2 r}$-term must be a boundary of the form $d^{2 r}\left(y^{n+1-r} \cdot w\right)$, with $w$ on the vertical axis. If $n \geq r$ then it follows that $z=d^{2 r}\left(y^{n-r} \cdot w\right)$ in the $E^{2 r}$-term, since by induction there is no $y$-torsion in filtration $-2 n$ of the $E^{2 r}$-term. Thus the only $y$-torsion in the $E^{2 r+2}$-term lies in filtrations $-2 r+2 \leq s \leq 0$.

In Section 7 we shall remark on an analogous homological Tate spectral sequence, where $P(y)$ is replaced by $P\left(y, y^{-1}\right)$ and the issue of $y$-torsion classes becomes void.

\section{$5 \quad$ Infinite cycles}

The Dyer-Lashof operations satisfy instability conditions [BMMS86, III.1.1] that are in a sense dual to those of the Steenrod operations. For a class $x \in$ $H_{t}\left(R ; \mathbb{F}_{p}\right)$ the lowest nontrivial operation is $Q^{t}(x)=x^{2}$ when $p=2, Q^{m}(x)=$ $x^{p}$ when $p$ is odd and $t=2 m$ is even, and $\beta Q^{m}(x)$ when $p$ is odd and $t=2 m-1$ is odd. Similarly, the lowest nontrivial operation on $\delta x \in H_{t+2 r-1}\left(R ; \mathbb{F}_{p}\right)$ with $d^{2 r}(x)=y^{r} \cdot \delta x$ is $Q^{t+2 r-1}(\delta x)=(\delta x)^{2}$ when $p=2, \beta Q^{m+r}(\delta x)$ when $p$ is odd and $t=2 m$ is even, and $Q^{m+r-1}(\delta x)=(\delta x)^{p}$ when $p$ is odd and $t=2 m-1$ is odd. Thus there is in each case a sequence of $(2 r-1)$ Dyer-Lashof operations $\beta^{\epsilon} Q^{i}$ whose action on $x$ can be nontrivial, but whose action on $\delta x$ must be trivial. By Proposition 4.2 this sequence of operations on $x$ will survive past the $E^{2 r}$-term, at least to the $E^{2 r+2}$-term. It is the main point of the present article to show that these classes, and one more "companion class", then in fact go on indefinitely to survive to the $E^{\infty}$-term, i.e., are infinite cycles! 
Theorem 5.1 Let $R$ be a $\mathbb{T}$-equivariant commutative $S$-algebra, suppose that $x \in H_{t}\left(R ; \mathbb{F}_{p}\right)$ survives to the $E^{2 r}$-term $E_{0, t}^{2 r} \subset H_{t}\left(R ; \mathbb{F}_{p}\right)$ of the homological homotopy fixed point spectral sequence for $R$, and write $d^{2 r}(x)=y^{r} \cdot \delta x$.

(a) For $p=2$, the $2 r$ classes

$$
x^{2}=Q^{t}(x), Q^{t+1}(x), \ldots, Q^{t+2 r-2}(x) \quad \text { and } Q^{t+2 r-1}(x)+x \delta x
$$

all survive to the $E^{\infty}$-term, i.e., are infinite cycles.

(b) For $p$ odd and $t=2 m$ even, the $2 r$ classes

$$
x^{p}=Q^{m}(x), \beta Q^{m+1}(x), \ldots, Q^{m+r-1}(x) \text { and } x^{p-1} \delta x
$$

all survive to the $E^{\infty}$-term, i.e., are infinite cycles.

(c) For $p$ odd and $t=2 m-1$ odd, the $2 r$ classes

$$
\beta Q^{m}(x), Q^{m}(x), \ldots, \beta Q^{m+r-1}(x) \text { and } Q^{m+r-1}(x)-x(\delta x)^{p-1}
$$

all survive to the $E^{\infty}$-term, i.e., are infinite cycles.

Proof The argument proceeds by considering a universal example. Recall that a class $x \in E_{0, t}^{2 r}$ is represented by a $\mathbb{T}$-equivariant map $x: S(\mathbb{C})_{+} \wedge S^{t} \rightarrow H \wedge R$ that admits an equivariant extension $x^{\prime}: S\left(\mathbb{C}^{r}\right)_{+} \wedge S^{t} \rightarrow H \wedge R$. Let

$$
X=D_{p}\left(S\left(\mathbb{C}^{r}\right)_{+} \wedge S^{t}\right)=E \Sigma_{p} \ltimes_{\Sigma_{p}}\left(S\left(\mathbb{C}^{r}\right)_{+} \wedge S^{t}\right)^{\wedge p}
$$

be the $p$-th extended power of the spectrum $S\left(\mathbb{C}^{r}\right)_{+} \wedge S^{t}$.

Somewhat abusively, we write $H_{*}\left(S\left(\mathbb{C}^{r}\right)_{+} \wedge S^{t} ; \mathbb{F}_{p}\right)=\mathbb{F}_{p}\{x, \delta x\}$ with $|x|=t$ and $|\delta x|=t+2 r-1$. Then the homology of the $p$-th extended power spectrum is

$$
H_{*}\left(X ; \mathbb{F}_{2}\right)=\mathbb{F}_{2}\left\{x \delta x, Q^{i}(x)\left|i \geq t, Q^{i}(\delta x)\right| i \geq t+2 r-1\right\}
$$

for $p=2$,

$$
H_{*}\left(X ; \mathbb{F}_{p}\right)=\mathbb{F}_{p}\left\{x^{p-1} \delta x, \beta^{\epsilon} Q^{i}(x)\left|i \geq m+\epsilon, \beta^{\epsilon} Q^{i}(\delta x)\right| i \geq m+r\right\}
$$

for $p$ odd and $t=2 m$ even, and

$$
H_{*}\left(X ; \mathbb{F}_{p}\right)=\mathbb{F}_{p}\left\{x(\delta x)^{p-1}, \beta^{\epsilon} Q^{i}(x)\left|i \geq m, \beta^{\epsilon} Q^{i}(\delta x)\right| i \geq m+r-1+\epsilon\right\}
$$

for $p$ odd and $t=2 m-1$ odd. Throughout $i$ is an integer and $\epsilon \in\{0,1\}$.

The equivariant extension $x^{\prime}$ induces an equivariant map

$$
D_{p}\left(x^{\prime}\right): X=D_{p}\left(S\left(\mathbb{C}^{r}\right)_{+} \wedge S^{t}\right) \rightarrow D_{p}(H \wedge R) .
$$


The $\mathbb{T}$-equivariant commutative $S$-algebra structures on $H$ and $R$ combine to form one on $H \wedge R$, and, as noted in Section 4, this gives $H \wedge R$ an $H_{\infty}$ ring structure ([EKMM97, Thm. 0.1, II.4 and III.5] and [LMS86, VII.2]). The associated $H_{\infty}$ structure includes, in particular, a $\mathbb{T}$-equivariant structure map

$$
\xi_{p}: D_{p}(H \wedge R) \rightarrow H \wedge R
$$

that extends the $p$-fold multiplication map on $H \wedge R$. Taken together, these produce an equivariant map

$H \wedge D_{p}\left(S\left(\mathbb{C}^{r}\right)_{+} \wedge S^{t}\right) \stackrel{1 \wedge D_{p}\left(x^{\prime}\right)}{\longrightarrow} H \wedge D_{p}(H \wedge R) \stackrel{1 \wedge \xi_{p}}{\longrightarrow} H \wedge H \wedge R \stackrel{\mu \wedge 1}{\longrightarrow} H \wedge R$,

where $\mu$ is the multiplication on $H$. Applying homotopy we have a homomorphism

$$
H_{*}\left(X ; \mathbb{F}_{p}\right)=H_{*}\left(D_{p}\left(S\left(\mathbb{C}^{r}\right)_{+} \wedge S^{t}\right) ; \mathbb{F}_{p}\right) \rightarrow H_{*}\left(R ; \mathbb{F}_{p}\right)
$$

which, by definition, takes the classes generating $H_{*}\left(X ; \mathbb{F}_{p}\right)$ to the classes with the same names in $H_{*}\left(R ; \mathbb{F}_{p}\right)$. Now $X=D_{p}\left(S\left(\mathbb{C}^{r}\right)_{+} \wedge S^{t}\right)$ is a $\mathbb{T}$-equivariant retract of the free commutative $S$-algebra

$$
P \simeq \bigvee_{j \geq 0} D_{j}\left(S\left(\mathbb{C}^{r}\right)_{+} \wedge S^{t}\right)
$$

on the space $S\left(\mathbb{C}^{r}\right)_{+} \wedge S^{t}$, so the homological homotopy fixed point spectral sequence for $X$ is a direct summand of the one for $P$. Thus the formula from Proposition 4.2 for the $d^{2 r}$-differentials in the spectral sequence for $P$ is also applicable in the spectral sequence for $X$.

Now consider the homological homotopy fixed point spectral sequence for $X=$ $D_{p}\left(S\left(\mathbb{C}^{r}\right)_{+} \wedge S^{t}\right)$, first for $p=2$ and then for $p$ odd. We shall show in each case that the $2 r$ classes in $E_{0, *}^{2 r} \subset H_{*}\left(X ; \mathbb{F}_{p}\right)$, with names as listed in the statement of the theorem, are infinite cycles. By naturality of the homotopy fixed point spectral sequence with respect to the map $H \wedge X \rightarrow H \wedge R$ from (5.1), it follows that the $2 r$ target classes listed in $E_{0, *}^{2 r} \subset H_{*}\left(R ; \mathbb{F}_{p}\right)$ are also infinite cycles. This will complete the proof of the theorem.

(a) Let $p=2$. The homological homotopy fixed point spectral sequence for $X$ has

$$
E_{* *}^{2}=P(y) \otimes \mathbb{F}_{2}\left\{x \delta x, Q^{i}(x)\left|i \geq t, Q^{i}(\delta x)\right| i \geq t+2 r-1\right\}
$$

and nontrivial differentials $d^{2 r}(x \delta x)=y^{r} \cdot(\delta x)^{2}$ and

$$
d^{2 r}\left(Q^{i}(x)\right)=y^{r} \cdot Q^{i}(\delta x)
$$

for all $i \geq t+2 r-1$, together with their $y$-multiples. 


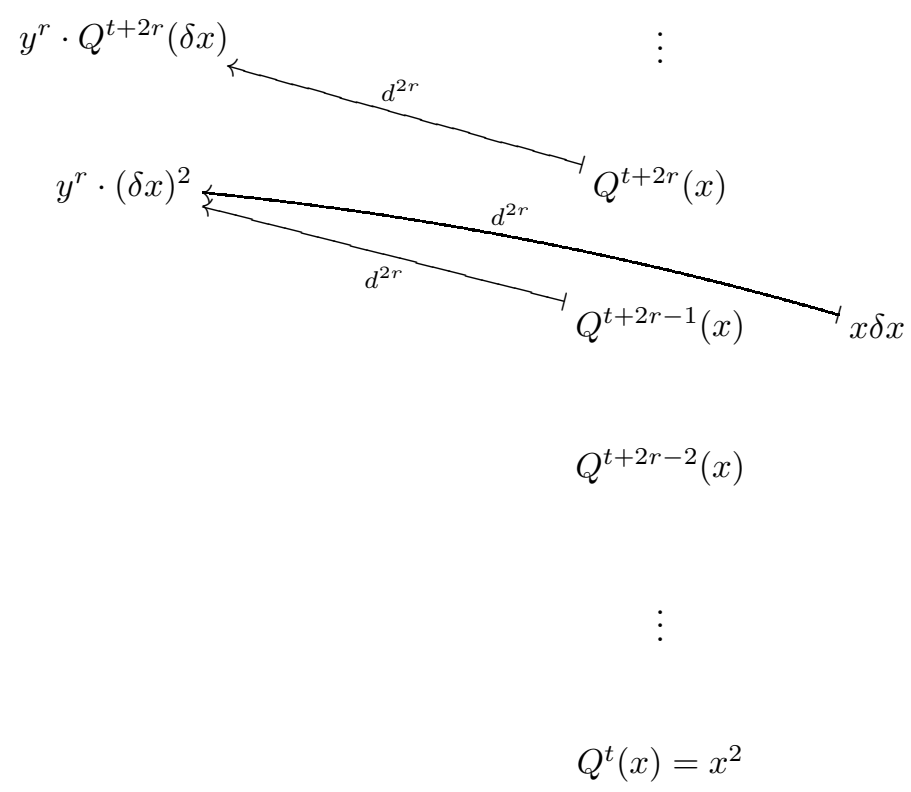

Figure 1: The case $p=2$

This leaves

$$
E_{* *}^{2 r+2}=P(y) \otimes \mathbb{F}_{2}\left\{Q^{i}(x) \mid t \leq i<t+2 r-1, Q^{t+2 r-1}(x)+x \delta x\right\}
$$

plus some $y$-torsion classes from $E_{* *}^{2}$ in filtrations $-2 r<s \leq 0$. Hence there are no classes remaining in the entire quadrant with filtration $s \leq-2 r$ and vertical degree $*>|x \delta x|=2 t+2 r-1$. All further differentials on the classes in $E_{0, *}^{2 r+2}$ on the vertical axis land in this zero region, since already $E_{0, *}^{2}$ starts in degree $2 t$ with the lowest class $Q^{t}(x)=x^{2}$. Thus all further differentials from the vertical axis are zero, and the spectral sequence collapses at $E_{* *}^{2 r+2}=E_{* *}^{\infty}$.

(b) Let $p$ be odd and $t=2 m$ even. The homological homotopy fixed point spectral sequence for $X$ has

$$
E_{* *}^{2}=P(y) \otimes \mathbb{F}_{p}\left\{x^{p-1} \delta x, \beta^{\epsilon} Q^{i}(x)\left|i \geq m+\epsilon, \beta^{\epsilon} Q^{i}(\delta x)\right| i \geq m+r\right\}
$$

and nontrivial differentials

$$
d^{2 r}\left(\beta^{\epsilon} Q^{i}(x)\right)=y^{r} \cdot \beta^{\epsilon} Q^{i}(\delta x)
$$

for $i \geq m+r$. This leaves

$$
E_{* *}^{2 r+2}=P(y) \otimes \mathbb{F}_{p}\left\{x^{p-1} \delta x, Q^{i}(x) \mid m+\epsilon \leq i<m+r\right\}
$$


plus some $y$-torsion classes in filtrations $-2 r<s \leq 0$. Hence there are no classes left in the region where $s \leq-2 r$ and the vertical degree is $*>$ $\left|Q^{m+r-1}(x)\right|$.

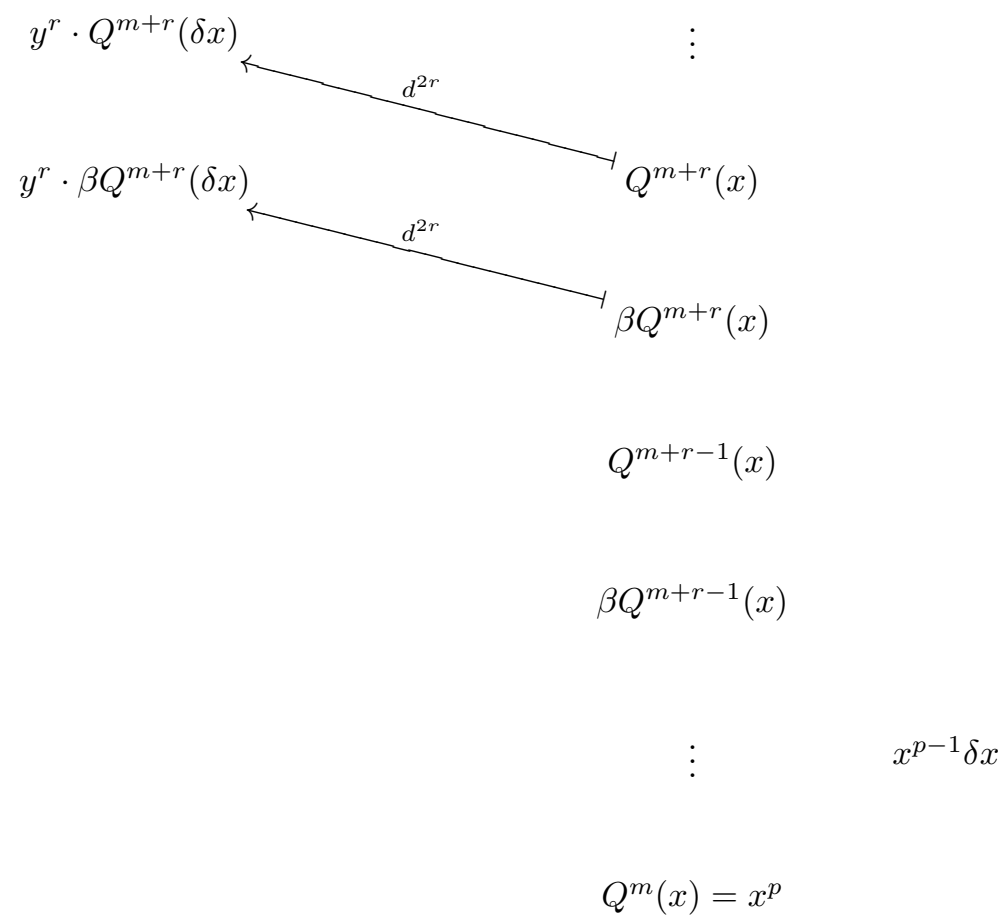

Figure 2: The case $p$ odd and $t=2 m$ even

Now, $x$ was also a class in the $E^{2 r-2}$-term, with $d^{2 r-2}(x)=0$, so by induction over $r$ we may assume (by naturality from the case of $(r-1)$ ) that the classes $\beta^{\epsilon} Q^{i}(x)$ with $m+\epsilon \leq i<m+(r-1)$ are infinite cycles. This leaves the three classes $x^{p-1} \delta x, \beta Q^{m+r-1}(x)$ and $Q^{m+r-1}(x)$ in $E_{0, *}^{2 r+2}$ that are not $y$-torsion, and could therefore imaginably support a differential after $d^{2 r}$. But the first two classes $\beta Q^{m+r-1}(x)$ and $Q^{m+r-1}(x)$ are so close to the horizontal edge of the vanishing region that all differentials after $d^{2 r}$ must vanish on these classes.

The third class $x^{p-1} \delta x$ has odd degree, so an even length differential on it must land in an even degree. The only even degree classes in filtrations $s \leq-2 r$ are the $y$-multiples of $Q^{i}(x)$ for $m \leq i<m+r$, of which $Q^{m}(x)=x^{p}$ is in lower degree than that of $x^{p-1} \delta x$. The remaining possible target classes $Q^{i}(x)$ for $m<i<m+r$ all have nontrivial Bockstein images $\beta Q^{i}(x)$, but 
$\beta\left(x^{p-1} \delta x\right)=0$ in $H_{*}\left(X ; \mathbb{F}_{p}\right)$. Therefore, by naturality of the differential with respect to the Bockstein operation, all of these targets for a differential on $x^{p-1} \delta x$ are excluded. Thus also $x^{p-1} \delta x$ is an infinite cycle.

(c) Let $p$ be odd and $t=2 m-1$ odd. The homological homotopy fixed point spectral sequence for $X$ has

$$
E_{* *}^{2}=P(y) \otimes \mathbb{F}_{p}\left\{x(\delta x)^{p-1}, \beta^{\epsilon} Q^{i}(x)\left|i \geq m, \beta^{\epsilon} Q^{i}(\delta x)\right| i \geq m+r-1+\epsilon\right\}
$$

and nontrivial differentials $d^{2 r}\left(x(\delta x)^{p-1}\right)=y^{r} \cdot(\delta x)^{p}$ and

$$
d^{2 r}\left(\beta^{\epsilon} Q^{i}(x)\right)=y^{r} \cdot \beta^{\epsilon} Q^{i}(\delta x)
$$

for $i \geq m+r-1+\epsilon$. This leaves

$E_{* *}^{2 r+2}=P(y) \otimes \mathbb{F}_{p}\left\{\beta^{\epsilon} Q^{i}(x) \mid m \leq i<m+r-1+\epsilon, Q^{m+r-1}(x)-x(\delta x)^{p-1}\right\}$

plus $y$-torsion classes in filtrations $-2 r<s \leq 0$. Hence there are no classes left in the region where $s \leq-2 r$ and the vertical degree is $*>\left|Q^{m+r-1}(x)\right|$.

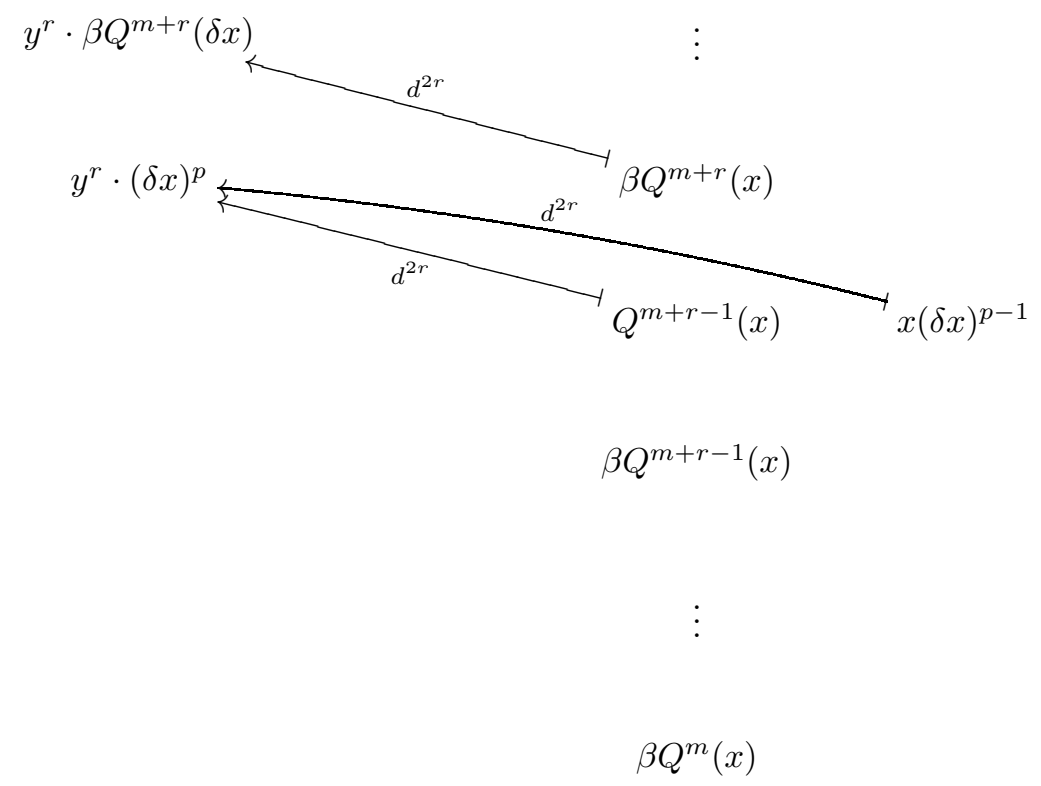

Figure 3: The case $p$ odd and $t=2 m-1$ odd

Again considering $x$ as a class in $E_{0, *}^{2 r-2}$ and using induction on $r$ we may assume that the classes $\beta^{\epsilon} Q^{i}(x)$ for $m \leq i<m+r-2+\epsilon$ and $Q^{m+r-2}(x)-x\left(\delta^{\prime} x\right)^{p-1}$ are infinite cycles. Here $\delta^{\prime} x$ is defined by $d^{2 r-2}(x)=y^{r-1} \cdot \delta^{\prime} x$. The fact that $d^{2 r-2}(x)=0$ gives $\delta^{\prime} x=0$, so in fact all the classes $\beta^{\epsilon} Q^{i}(x)$ for $m \leq i<$ $m+r-1$ in $E_{* *}^{2 r+2}$ are infinite cycles. 
This leaves only the two classes $\beta Q^{m+r-1}(x)$ and $Q^{m+r-1}(x)-x(\delta x)^{p-1}$, but these are so close to the horizontal border of the vanishing region that all differentials after $d^{2 r}$ must be zero on them.

\section{Examples}

Our Theorem 5.1 has applications to the homological homotopy fixed point spectral sequence for the commutative $S$-algebra $R=T H H(B)$ given by the topological Hochschild homology of a commutative $S$-algebra $B$. The $\mathbb{T}$ homotopy fixed point spectrum $T H H(B)^{h \mathbb{T}}$ is closely related to the topological model $T F(B)$ for the negative cyclic homology of $B$, which in turn is very close to the topological cyclic homology $T C(B)$ BHM93 and algebraic $K$ theory $K(B)$ of $B$ [Du97. These spectral sequences therefore have significant interest.

First consider the connective Johnson-Wilson spectrum $B=B P\langle m-1\rangle$, for some prime $p$ and integer $0 \leq m<\infty$. So

$$
\pi_{*} B P\langle m-1\rangle=B P_{*} /\left(v_{n} \mid n \geq m\right),
$$

where $B P_{*}=\mathbb{Z}_{(p)}\left[v_{n} \mid n \geq 1\right]$ and $v_{0}=p$, and

$$
H_{*}\left(B P\langle m-1\rangle ; \mathbb{F}_{p}\right)= \begin{cases}P\left(\bar{\xi}_{1}^{2}, \ldots, \bar{\xi}_{m}^{2}, \bar{\xi}_{m+1}, \ldots\right) & \text { for } p=2, \\ P\left(\bar{\xi}_{k} \mid k \geq 1\right) \otimes E\left(\bar{\tau}_{k} \mid k \geq m\right) & \text { for } p \text { odd }\end{cases}
$$

The latter is a sub-algebra of the dual Steenrod algebra $A_{*}=H_{*}\left(H \mathbb{F}_{p} ; \mathbb{F}_{p}\right)$.

Suppose that $p$ and $m$ are such that $B P\langle m-1\rangle$ admits the structure of a commutative $S$-algebra. This is so at least for $m \in\{0,1,2\}$, when $B P\langle-1\rangle=$ $H \mathbb{F}_{p}, B P\langle 0\rangle=H \mathbb{Z}_{(p)}$ and $B P\langle 1\rangle=\ell$, respectively, where $\ell$ is the Adams summand of $p$-local connective topological $K$-theory $k u_{(p)}$. When $p=2$, $\ell=k u_{(2)}$.

Then the Bökstedt spectral sequence

$$
E_{* *}^{2}=H H_{*}\left(H_{*}\left(B ; \mathbb{F}_{p}\right)\right) \Longrightarrow H_{*}\left(T H H(B) ; \mathbb{F}_{p}\right)
$$

has $E^{2}$-term

$$
E_{* *}^{2}= \begin{cases}H_{*}\left(B P\langle m-1\rangle ; \mathbb{F}_{2}\right) \otimes E\left(\sigma \bar{\xi}_{1}^{2}, \ldots, \sigma \bar{\xi}_{m}^{2}, \sigma \bar{\xi}_{m+1}, \ldots\right) & \text { for } p=2, \\ H_{*}\left(B P\langle m-1\rangle ; \mathbb{F}_{p}\right) \otimes E\left(\sigma \bar{\xi}_{k} \mid k \geq 1\right) \otimes \Gamma\left(\sigma \bar{\tau}_{k} \mid k \geq m\right) & \text { for } p \text { odd }\end{cases}
$$

For $x \in H_{*}\left(B ; \mathbb{F}_{p}\right), \sigma x \in H H_{1}\left(H_{*}\left(B ; \mathbb{F}_{p}\right)\right)$ is represented by the Hochschild 1 -cycle $1 \otimes x$. The operator $\sigma$ is a differential $\left(\sigma^{2}=0\right)$ and a graded derivation $\left(\sigma(x y)=x \sigma(y)+(-1)^{|y|} \sigma(x) y\right)$. Here $\Gamma(-)$ denotes the divided power algebra. 
For $p$ odd, Bökstedt found differentials

$$
d^{p-1}\left(\gamma_{j}\left(\sigma \bar{\tau}_{k}\right)\right)=\sigma \bar{\xi}_{k+1} \cdot \gamma_{j-p}\left(\sigma \bar{\tau}_{k}\right)
$$

for $j \geq p$, and in all cases the spectral sequence collapses at the $E^{p}$-term. So

$$
E_{* *}^{\infty}= \begin{cases}H_{*}\left(B P\langle m-1\rangle ; \mathbb{F}_{2}\right) \otimes E\left(\sigma \bar{\xi}_{1}^{2}, \ldots, \sigma \bar{\xi}_{m}^{2}, \sigma \bar{\xi}_{m+1}, \ldots\right) & \text { for } p=2, \\ H_{*}\left(B P\langle m-1\rangle ; \mathbb{F}_{p}\right) \otimes E\left(\sigma \bar{\xi}_{1}, \ldots, \sigma \bar{\xi}_{m}\right) \otimes P_{p}\left(\sigma \bar{\tau}_{k} \mid k \geq m\right) & \text { for } p \text { odd }\end{cases}
$$

Here $P_{p}(-)$ denotes the truncated polynomial algebra of height $p$.

If $B P\langle m-1\rangle$, and thus $T H H(B P\langle m-1\rangle)$, is a commutative $S$-algebra, then there are multiplicative extensions $\left(\sigma \bar{\xi}_{k}\right)^{2}=\sigma \bar{\xi}_{k+1}$ for $p=2$ and $\left(\sigma \bar{\tau}_{k}\right)^{p}=$ $\sigma \bar{\tau}_{k+1}$ for $p$ odd, so

$$
\begin{aligned}
& H_{*}\left(T H H(B P\langle m-1\rangle) ; \mathbb{F}_{p}\right) \\
& \quad= \begin{cases}H_{*}\left(B P\langle m-1\rangle ; \mathbb{F}_{2}\right) \otimes E\left(\sigma \bar{\xi}_{1}^{2}, \ldots, \sigma \bar{\xi}_{m}^{2}\right) \otimes P\left(\sigma \bar{\xi}_{m+1}\right) & \text { for } p=2, \\
H_{*}\left(B P\langle m-1\rangle ; \mathbb{F}_{p}\right) \otimes E\left(\sigma \bar{\xi}_{1}, \ldots, \sigma \bar{\xi}_{m}\right) \otimes P\left(\sigma \bar{\tau}_{m}\right) & \text { for } p \text { odd } .\end{cases}
\end{aligned}
$$

For more references and details on the calculation up to this point, see $\mathrm{AnR}$, Ch. 5].

We now consider the homological homotopy fixed point spectral sequence for $R=T H H(B)$. It starts with

$$
E_{* *}^{2}=P(y) \otimes H_{*}\left(T H H(B) ; \mathbb{F}_{p}\right)
$$

and by Lemma 3.1 it has first differentials

$$
d^{2}(x)=y \cdot \sigma x
$$

for all $x \in H_{*}\left(T H H(B) ; \mathbb{F}_{p}\right)$. Here $\sigma x \in H_{t+1}\left(T H H(B) ; \mathbb{F}_{p}\right)$ is the image of $x \otimes s_{1} \in H_{t}\left(T H H(B) ; \mathbb{F}_{p}\right) \otimes H_{1}\left(\mathbb{T} ; \mathbb{F}_{p}\right)$ under the circle action map

$$
\alpha: \operatorname{THH}(B) \wedge \mathbb{T}_{+} \rightarrow \operatorname{THH}(B),
$$

where $s_{1} \in H_{1}\left(\mathbb{T} ; \mathbb{F}_{p}\right)$ is the canonical generator. By Lemma 4.3(a) we have similar differentials $d^{2}\left(y^{n} \cdot x\right)=y^{n+1} \cdot \sigma x$ for all $n \geq 0$.

Hence we can find the columns of $E_{* *}^{4}$ in the homological homotopy fixed point spectral sequence by passing to the homology of $E_{0, *}^{2}=H_{*}\left(T H H(B) ; \mathbb{F}_{p}\right)$ with respect to the operator $\sigma$, at least to the left of the vertical axis.

Proposition 6.1 The homological homotopy fixed point spectral sequence for $R=T H H(B)$ with $B=B P\langle m-1\rangle$, for $p$ and $m$ such that $B$ is a commutative $S$-algebra, collapses after the $d^{2}$-differentials, with the following $E^{\infty}$-term: 
(a) For $p=2$,

$$
E_{* *}^{\infty}=P(y) \otimes P\left(\bar{\xi}_{1}^{4}, \ldots, \bar{\xi}_{m}^{4}, \bar{\xi}_{m+1}^{2}, \xi_{m+2}^{\prime}, \ldots\right) \otimes E\left(\bar{\xi}_{1}^{2} \sigma \bar{\xi}_{1}^{2}, \ldots, \bar{\xi}_{m}^{2} \sigma \bar{\xi}_{m}^{2}\right)
$$

plus some classes in filtration $s=0$, where $\xi_{k+1}^{\prime}=\bar{\xi}_{k+1}+\bar{\xi}_{k} \sigma \bar{\xi}_{k}$ for $k \geq m+1$.

(b) For $p$ odd,

$$
\begin{aligned}
E_{* *}^{\infty}=P(y) \otimes P\left(\bar{\xi}_{k}^{p} \mid 1 \leq\right. & k \leq m) \otimes P\left(\bar{\xi}_{k+1} \mid k \geq m\right) \\
& \otimes E\left(\tau_{k+1}^{\prime} \mid k \geq m\right) \otimes E\left(\bar{\xi}_{k}^{p-1} \sigma \bar{\xi}_{k} \mid 1 \leq k \leq m\right)
\end{aligned}
$$

plus some classes in filtration $s=0$, where $\tau_{k+1}^{\prime}=\bar{\tau}_{k+1}-\bar{\tau}_{k}\left(\sigma \bar{\tau}_{k}\right)^{p-1}$ for $k \geq m$.

Proof (a) For $B=B P\langle m-1\rangle$ and $p=2$ we have

$$
\begin{aligned}
E_{0, *}^{2}=H_{*} & \left(T H H(B P\langle m-1\rangle) ; \mathbb{F}_{2}\right) \\
= & P\left(\bar{\xi}_{1}^{2}, \ldots, \bar{\xi}_{m}^{2}, \bar{\xi}_{m+1}, \ldots\right) \otimes E\left(\sigma \bar{\xi}_{1}^{2}, \ldots, \sigma \bar{\xi}_{m}^{2}\right) \otimes P\left(\sigma \bar{\xi}_{m+1}\right) .
\end{aligned}
$$

Here $\sigma: \bar{\xi}_{k}^{2} \mapsto \sigma \bar{\xi}_{k}^{2}$ for $1 \leq k \leq m$ and $\sigma: \bar{\xi}_{k+1} \mapsto \sigma \bar{\xi}_{k+1}$ for $k \geq m$. We have $\sigma \bar{\xi}_{k+1}=\left(\sigma \bar{\xi}_{k}\right)^{2}$ for $k \geq m+1$.

So the squares $\left(\bar{\xi}_{k}^{2}\right)^{2}=\bar{\xi}_{k}^{4}$ and $\bar{\xi}_{m+1}^{2}$, as well as the companion classes defined by

$$
\xi_{k+1}^{\prime}=\bar{\xi}_{k+1}+\bar{\xi}_{k} \sigma \bar{\xi}_{k}
$$

for $k \geq m+1$, are $d^{2}$-cycles, while $E\left(\bar{\xi}_{k}^{2}, \sigma \bar{\xi}_{k}^{2}\right)$ has homology $E\left(\bar{\xi}_{k}^{2} \sigma \bar{\xi}_{k}^{2}\right)$ for each $k$, and $E\left(\bar{\xi}_{m+1}\right) \otimes P\left(\sigma \bar{\xi}_{m+1}\right)$ has homology $\mathbb{F}_{2}$.

Hence the homological spectral sequence has

$$
E_{* *}^{4}=P(y) \otimes P\left(\bar{\xi}_{1}^{4}, \ldots, \bar{\xi}_{m}^{4}, \bar{\xi}_{m+1}^{2}, \xi_{m+2}^{\prime}, \ldots\right) \otimes E\left(\bar{\xi}_{1}^{2} \sigma \bar{\xi}_{1}^{2}, \ldots, \bar{\xi}_{m}^{2} \sigma \bar{\xi}_{m}^{2}\right)
$$

plus the image of $\sigma$ in filtration $s=0$.

By our Theorem [5.1(a) applied to the classes $x=\bar{\xi}_{k}^{2}$ for $1 \leq k \leq m$, in even degree $t=|x|$, the classes $x^{2}=\bar{\xi}_{k}^{4}$ and $Q^{t+1}(x)+x \sigma x=\overline{\bar{\xi}}_{k}^{2} \sigma \bar{\xi}_{k}^{2}$ are infinite cycles, for $Q^{t+1}\left(\bar{\xi}_{k}^{2}\right)=0$ by the Cartan formula.

Similarly, by Theorem[5.1(a) applied to the classes $x=\bar{\xi}_{k}$ for $k \geq m+1$, in odd degree $t=|x|$, the classes $x^{2}=\bar{\xi}_{k}^{2}$ and $Q^{t+1}(x)+x \sigma x=\bar{\xi}_{k+1}+\bar{\xi}_{k} \sigma \bar{\xi}_{k}=\xi_{k+1}^{\prime}$ are infinite cycles. For $Q^{t+1}\left(\bar{\xi}_{k}\right)=\bar{\xi}_{k+1}$ by [BMMS86, III.2.2 and I.3.6].

The extra classes in filtration $s=0$ are $y$-torsion, hence infinite cycles. Therefore the $E^{4}$-term above is generated as an algebra by infinite cycles, so the homological spectral sequence collapses at this stage. 
(b) For $B=B P\langle m-1\rangle$ and $p$ odd we have

$$
\begin{aligned}
& E_{0, *}^{2}=H_{*}\left(T H H(B P\langle m-1\rangle) ; \mathbb{F}_{p}\right) \\
& \quad=P\left(\bar{\xi}_{k} \mid k \geq 1\right) \otimes E\left(\bar{\tau}_{k} \mid k \geq m\right) \otimes E\left(\sigma \bar{\xi}_{k} \mid 1 \leq k \leq m\right) \otimes P\left(\sigma \bar{\tau}_{m}\right) .
\end{aligned}
$$

Here $\sigma: \bar{\xi}_{k} \mapsto \sigma \bar{\xi}_{k}$ for $1 \leq k \leq m, \sigma: \bar{\xi}_{k+1} \mapsto 0$ for $k \geq m$ and $\sigma: \bar{\tau}_{k} \mapsto \sigma \bar{\tau}_{k}$ for $k \geq m$. We have $\sigma \bar{\tau}_{k+1}=\left(\sigma \bar{\tau}_{k}\right)^{p}$ for $k \geq m$.

So the $p$-th powers $\bar{\xi}_{k}^{p}$ for $1 \leq k \leq m$, the classes $\bar{\xi}_{k+1}$ for $k \geq m$, and the companion classes defined by

$$
\tau_{k+1}^{\prime}=\bar{\tau}_{k+1}-\bar{\tau}_{k}\left(\sigma \bar{\tau}_{k}\right)^{p-1}
$$

for $k \geq m$, are $d^{2}$-cycles, while $P_{p}\left(\bar{\xi}_{k}\right) \otimes E\left(\sigma \bar{\xi}_{k}\right)$ has homology $E\left(\bar{\xi}_{k}^{p-1} \sigma \bar{\xi}_{k}\right)$ for each $1 \leq k \leq m$, and $E\left(\bar{\tau}_{m}\right) \otimes P\left(\sigma \bar{\tau}_{m}\right)$ has homology $\mathbb{F}_{p}$.

Hence the homological spectral sequence has

$$
\begin{aligned}
E_{* *}^{4}=P(y) \otimes P\left(\bar{\xi}_{k}^{p} \mid 1 \leq\right. & k \leq m) \otimes P\left(\bar{\xi}_{k+1} \mid k \geq m\right) \\
& \otimes E\left(\tau_{k+1}^{\prime} \mid k \geq m\right) \otimes E\left(\bar{\xi}_{k}^{p-1} \sigma \bar{\xi}_{k} \mid 1 \leq k \leq m\right)
\end{aligned}
$$

plus some classes in filtration $s=0$.

Applying our Theorem [5.1(b) to the classes $x=\bar{\xi}_{k}$ for $1 \leq k \leq m$, in even degree $t=|x|$, the classes $x^{p}=\bar{\xi}_{k}^{p}$ and $x^{p-1} \sigma x=\bar{\xi}_{k}^{p-1} \sigma \bar{\xi}_{k}$ are infinite cycles.

Similarly, applying Theorem 5.11(c) to the classes $x=\bar{\tau}_{k}$ for $k \geq m$, in odd degree $t=|x|=2 p^{k}-1$, the classes $\beta Q^{p^{k}}(x)=\bar{\xi}_{k+1}$ and $Q^{p^{k}}(x)-x(\sigma x)^{p-1}=$ $\bar{\tau}_{k+1}-\bar{\tau}_{k}\left(\sigma \bar{\tau}_{k}\right)^{p-1}=\tau_{k+1}^{\prime}$ are infinite cycles, for $Q^{p^{k}}\left(\bar{\tau}_{k}\right)=\bar{\tau}_{k+1}$ and $\beta \bar{\tau}_{k+1}=$ $\bar{\xi}_{k+1}$ by BMMS86, III.2.3 and I.3.6].

Hence the $E^{4}$-term above is generated as an algebra by infinite cycles, and the homological spectral sequence collapses after the $d^{2}$-differentials.

For convenience in the comparison with $k o$, we make the case $B=k u$ at $p=2$ explicit:

Corollary 6.2 The homological homotopy fixed point spectral sequence for $R=T H H(k u)$ at $p=2$ collapses after the $d^{2}$-differentials, with

$$
E_{* *}^{\infty}=P(y) \otimes P\left(\bar{\xi}_{1}^{4}, \bar{\xi}_{2}^{4}, \bar{\xi}_{3}^{2}, \xi_{4}^{\prime}, \ldots\right) \otimes E\left(\bar{\xi}_{1}^{2} \sigma \bar{\xi}_{1}^{2}, \bar{\xi}_{2}^{2} \sigma \bar{\xi}_{2}^{2}\right)
$$

plus some classes in filtration $s=0$, where $\xi_{k+1}^{\prime}=\bar{\xi}_{k+1}+\bar{\xi}_{k} \sigma \bar{\xi}_{k}$ for $k \geq 3$. 
Proposition 6.3 The homological homotopy fixed point spectral sequence for $R=T H H(B)$ collapses after the $d^{2}$-differentials, in both of the cases:

(a) $B=k o$ and $p=2$, when

$$
E_{* *}^{\infty}=P(y) \otimes P\left(\bar{\xi}_{1}^{8}, \bar{\xi}_{2}^{4}, \bar{\xi}_{3}^{2}, \xi_{4}^{\prime}, \ldots\right) \otimes E\left(\bar{\xi}_{1}^{4} \sigma \bar{\xi}_{1}^{4}, \bar{\xi}_{2}^{2} \sigma \bar{\xi}_{2}^{2}\right)
$$

plus classes on the vertical axis, and

(b) $B=$ tmf and $p=2$, when

$$
E_{* *}^{\infty}=P(y) \otimes P\left(\bar{\xi}_{1}^{16}, \bar{\xi}_{2}^{8}, \bar{\xi}_{3}^{4}, \bar{\xi}_{4}^{2}, \xi_{5}^{\prime}, \ldots\right) \otimes E\left(\bar{\xi}_{1}^{8} \sigma \bar{\xi}_{1}^{8}, \bar{\xi}_{2}^{4} \sigma \bar{\xi}_{2}^{4}, \bar{\xi}_{3}^{2} \sigma \bar{\xi}_{3}^{2}\right)
$$

plus classes on the vertical axis.

Proof (a) For $B=k o$ with $H_{*}\left(B ; \mathbb{F}_{2}\right)=\left(A / / A_{1}\right)_{*}=P\left(\bar{\xi}_{1}^{4}, \bar{\xi}_{2}^{2}, \bar{\xi}_{3}, \ldots\right)$ we have

$$
H_{*}\left(T H H(k o) ; \mathbb{F}_{2}\right)=P\left(\bar{\xi}_{1}^{4}, \bar{\xi}_{2}^{2}, \bar{\xi}_{3}, \ldots\right) \otimes E\left(\sigma \bar{\xi}_{1}^{4}, \sigma \bar{\xi}_{2}^{2}\right) \otimes P\left(\sigma \bar{\xi}_{3}\right) .
$$

See $\underline{\mathrm{AnR}}, 6.2(\mathrm{a})]$.

As in the proof of Proposition 6.1 the squares $\bar{\xi}_{1}^{8}, \bar{\xi}_{2}^{4}$ and $\bar{\xi}_{3}^{2}$, as well as the classes $\xi_{k+1}^{\prime}=\bar{\xi}_{k+1}+\bar{\xi}_{k} \sigma \bar{\xi}_{k}$ for $k \geq 3$ are $d^{2}$-cycles, while $E\left(\bar{\xi}_{1}^{4}, \sigma \bar{\xi}_{1}^{4}\right)$ and $E\left(\bar{\xi}_{2}^{2}, \sigma \bar{\xi}_{2}^{2}\right)$ have homology $E\left(\bar{\xi}_{1}^{4} \sigma \bar{\xi}_{1}^{4}\right)$ and $E\left(\bar{\xi}_{2}^{2} \sigma \bar{\xi}_{2}^{2}\right)$, respectively. The homology of $E\left(\bar{\xi}_{3}\right) \otimes P\left(\sigma \bar{\xi}_{3}\right)$ is $\mathbb{F}_{2}$. So

$$
E_{* *}^{4}=P(y) \otimes P\left(\bar{\xi}_{1}^{8}, \bar{\xi}_{2}^{4}, \bar{\xi}_{3}^{2}, \xi_{4}^{\prime}, \ldots\right) \otimes E\left(\bar{\xi}_{1}^{4} \sigma \bar{\xi}_{1}^{4}, \bar{\xi}_{2}^{2} \sigma \bar{\xi}_{2}^{2}\right)
$$

plus some classes in filtration $s=0$.

By Theorem 5.1(a), all of these algebra generators are in fact infinite cycles, so the homological spectral sequence collapses, as claimed.

(b) For $B=$ tmf with $H_{*}\left(B ; \mathbb{F}_{2}\right)=\left(A / / A_{2}\right)_{*}=P\left(\bar{\xi}_{1}^{8}, \bar{\xi}_{2}^{4}, \bar{\xi}_{3}^{2}, \bar{\xi}_{4}, \ldots\right)$ we have

$$
H_{*}\left(T H H(t m f) ; \mathbb{F}_{2}\right)=P\left(\bar{\xi}_{1}^{8}, \bar{\xi}_{2}^{4}, \bar{\xi}_{3}^{2}, \bar{\xi}_{4}, \ldots\right) \otimes E\left(\sigma \bar{\xi}_{1}^{8}, \sigma \bar{\xi}_{2}^{4}, \sigma \bar{\xi}_{3}^{2}\right) \otimes P\left(\sigma \bar{\xi}_{4}\right) .
$$

See [AnR, 6.2(b)]. This gives the $E^{2}$-term of the homological spectral sequence, and as before its homology with respect to the $\sigma$-operator is

$$
E_{* *}^{4}=P(y) \otimes P\left(\bar{\xi}_{1}^{16}, \bar{\xi}_{2}^{8}, \bar{\xi}_{3}^{4}, \bar{\xi}_{4}^{2}, \xi_{5}^{\prime}, \ldots\right) \otimes E\left(\bar{\xi}_{1}^{8} \sigma \bar{\xi}_{1}^{8}, \bar{\xi}_{2}^{4} \sigma \bar{\xi}_{2}^{4}, \bar{\xi}_{3}^{2} \sigma \bar{\xi}_{3}^{2}\right)
$$

plus some classes in filtration $s=0$.

By Theorem 5.1(a), all of these algebra generators are in fact infinite cycles, so the homological spectral sequence collapses, as claimed. 
Theorem 6.4 The homological homotopy fixed point spectral sequence for $R=T H H(B)$ collapses after the $d^{2}$-differentials, in both of the cases:

(a) $B=M U$, with

$$
E_{* *}^{\infty}=P(y) \otimes P\left(b_{k}^{p} \mid k \geq 1\right) \otimes E\left(b_{k}^{p-1} \sigma b_{k} \mid k \geq 1\right)
$$

plus classes in filtration zero, and

(b) $B=B P$, with

$$
E_{* *}^{\infty}=P(y) \otimes P\left(\xi_{k}^{p} \mid k \geq 1\right) \otimes E\left(\xi_{k}^{p-1} \sigma \xi_{k} \mid k \geq 1\right)
$$

plus classes in filtration zero. (When $p=2$, substitute $\xi_{k}^{2}$ for $\xi_{k}$.)

Note that we do not need to assume that $B P$ is a commutative $S$-algebra for the result in part (b).

Proof (a) The integral homology algebra of $M U$ is $H_{*}(M U ; \mathbb{Z})=\mathbb{Z}\left[b_{k}\right.$ $k \geq 1$ ], where $b_{k}$ in degree $2 k$ is the stabilized image of the generator $\beta_{k+1} \in$ $H_{2 k+2}(B U(1) ; \mathbb{Z})$, under the zero-section identification $B U(1) \simeq M U(1)$. So

$$
H_{*}\left(M U ; \mathbb{F}_{p}\right)=P\left(b_{k} \mid k \geq 1\right)
$$

is concentrated in even degrees, and the $E^{2}$-term of the Bökstedt spectral sequence is

$$
E_{* *}^{2}=H H_{*}\left(H_{*}\left(M U ; \mathbb{F}_{p}\right)\right)=H_{*}\left(M U ; \mathbb{F}_{p}\right) \otimes E\left(\sigma b_{k} \mid k \geq 1\right) .
$$

All the algebra generators are in filtrations $s \leq 1$, so the spectral sequence collapses at this stage. There are no algebra extensions, since for $p=2$, $\left(\sigma b_{k}\right)^{2}=Q^{2 k+1}\left(\sigma b_{k}\right)=\sigma Q^{2 k+1}\left(b_{k}\right)=0$, where $Q^{2 k+1}\left(b_{k}\right)=0$ because it has odd degree. For $p$ odd, $\left(\sigma b_{k}\right)^{2}=0$ by graded commutativity, because $\sigma b_{k}$ has odd degree. Thus

$$
H_{*}\left(T H H(M U) ; \mathbb{F}_{p}\right)=H_{*}\left(M U ; \mathbb{F}_{p}\right) \otimes E\left(\sigma b_{k} \mid k \geq 1\right) .
$$

This much can also be read off from MS93, 4.3], or from Cohen and Schlichtkrull's formula $T H H(M U) \simeq M U \wedge S U_{+}$[CS.

The homological homotopy fixed point spectral sequence has $E^{2}$-term

$$
E_{* *}^{2}=P(y) \otimes P\left(b_{k} \mid k \geq 1\right) \otimes E\left(\sigma b_{k} \mid k \geq 1\right) .
$$

Its homology with respect to the $d^{2}$-differential, satisfying $d^{2}\left(b_{k}\right)=y \cdot \sigma b_{k}$, is

$$
E_{* *}^{4}=P(y) \otimes P\left(b_{k}^{p} \mid k \geq 1\right) \otimes E\left(b_{k}^{p-1} \sigma b_{k} \mid k \geq 1\right)
$$


plus the usual $y$-torsion on the vertical axis. By Theorem 5.1(a) and (b), the algebra generators of this $E^{4}$-term are all infinite cycles. Hence the spectral sequence collapses at this stage.

(b) The Brown-Peterson spectrum $B P$ was originally constructed to have mod $p$ homology

$$
H_{*}\left(B P ; \mathbb{F}_{p}\right)= \begin{cases}P\left(\xi_{k}^{2} \mid k \geq 1\right) & \text { for } p=2, \\ P\left(\xi_{k} \mid k \geq 1\right) & \text { for } p \text { odd }\end{cases}
$$

This equals the sub-algebra $(A / / E)_{*}$ of $A_{*}$ that is dual to the quotient algebra $A / / E=A / A \beta A$ of $A$. Hereafter we focus on the odd-primary case; the reader should substitute $\xi_{k}^{2}$ for $\xi_{k}$ when $p=2$.

The spectrum $B P$ is known to be an (associative) $S$-algebra, and to receive an $S$-algebra map from $M U$ [BJ02, 3.5]. This map induces a split surjective algebra homomorphism $H_{*}\left(M U ; \mathbb{F}_{p}\right) \rightarrow H_{*}\left(B P ; \mathbb{F}_{p}\right)$ in homology, which maps $b_{p^{k}-1}$ to $\xi_{k}$ for $k \geq 1$ and takes the remaining algebra generators $b_{i}$ to 0 for $i \neq p^{k}-1$. For the homology of $B P$ injects into $H_{*}\left(H \mathbb{Z}_{(p)} ; \mathbb{F}_{p}\right)$ and at the level of second spaces the composite map of spectra $M U \rightarrow B P \rightarrow$ $H \mathbb{Z}_{(p)}$ is a $p$-local equivalence $M U(1) \rightarrow K\left(\mathbb{Z}_{(p)}, 2\right)$. The generator $\beta_{i+1} \in$ $\tilde{H}_{2 i+2}\left(M U(1) ; \mathbb{F}_{p}\right)$ maps to $b_{i} \in H_{2 i}\left(M U ; \mathbb{F}_{p}\right)$, while the corresponding generator $\beta_{i+1} \in \tilde{H}_{2 i+2}\left(K\left(\mathbb{Z}_{(p)}, 2\right) ; \mathbb{F}_{p}\right)$ maps to $\xi_{k} \in H_{2 i}\left(H \mathbb{Z}_{(p)} ; \mathbb{F}_{p}\right)$ when $i=p^{k}-1$ and to 0 otherwise [Mi58, Ch. 5]. This proves the claim.

The Bökstedt spectral sequence for $B P$ has $E^{2}$-term

$$
E_{* *}^{2}=H H_{*}\left(H_{*}\left(B P ; \mathbb{F}_{p}\right)\right)=H_{*}\left(B P ; \mathbb{F}_{p}\right) \otimes E\left(\sigma \xi_{k} \mid k \geq 1\right) .
$$

Note that the map $M U \rightarrow B P$ induces a surjection of Bökstedt spectral sequence $E^{2}$-terms. Thus the fact that the Bökstedt spectral sequence for $M U$ collapses at $E^{2}$ with no algebra extensions implies the corresponding statement for $B P$, also without the assumption that $B P$ is a commutative $S$-algebra. We can conclude that

$$
H_{*}\left(T H H(B P) ; \mathbb{F}_{p}\right)=H_{*}\left(B P ; \mathbb{F}_{p}\right) \otimes E\left(\sigma \xi_{k} \mid k \geq 1\right) .
$$

The homological homotopy fixed point spectral sequence has $E^{2}$-term

$$
E_{* *}^{2}=P(y) \otimes P\left(\xi_{k} \mid k \geq 1\right) \otimes E\left(\sigma \xi_{k} \mid k \geq 1\right) .
$$

Again the map $M U \rightarrow B P$ induces a surjection of $E^{2}$-terms, so the $d^{2}$ differentials satisfy $d^{2}\left(\xi_{k}\right)=y \cdot \sigma \xi_{k}$ and $d^{2}(y)=0$, and are derivations. This leaves

$$
E_{* *}^{4}=P(y) \otimes P\left(\xi_{k}^{p} \mid k \geq 1\right) \otimes E\left(\xi_{k}^{p-1} \sigma \xi_{k} \mid k \geq 1\right)
$$


plus some $y$-torsion on the vertical axis, and the map from the $E^{4}$-term of the spectral sequence for $M U$ is still surjective. Thus the spectral sequence for $B P$ also collapses at this stage.

\section{$7 \quad$ Generalizations and comments}

In this section we note some generalizations of our results, and also comment on the relation to similar patterns of differentials in other spectral sequences. The generalizations are of two sorts. First, we can replace the homotopy fixed points construction by the Tate construction or the homotopy orbits. Second, we can change the group of equivariance. We consider these in order.

First, there are spectral sequences similar to the one considered here for the Tate construction $\left.X^{t \mathbb{T}}=\widetilde{[E \mathbb{T}} \wedge F\left(E \mathbb{T}_{+}, X\right)\right]^{\mathbb{T}}$ (denoted $t_{\mathbb{T}}(X)^{\mathbb{T}}$ in GM95 and $\widehat{\mathbb{H}}(\mathbb{T}, X)$ in AuR02 $)$ and the homotopy orbit spectrum $X_{h \mathbb{T}}=E \mathbb{T}_{+} \wedge_{\mathbb{T}} X$.

Proposition 7.1 There is a natural spectral sequence

$$
\widehat{E}_{* *}^{2}=\widehat{H}^{-*}\left(\mathbb{T} ; H_{*}\left(X ; \mathbb{F}_{p}\right)\right)=P\left(y, y^{-1}\right) \otimes H_{*}\left(X ; \mathbb{F}_{p}\right)
$$

with $y$ in bidegree $(-2,0)$, which is conditionally convergent to the continuous homology $H_{*}^{c}\left(X^{t \mathbb{T}} ; \mathbb{F}_{p}\right)$. We call this the homological Tate spectral sequence. If $H_{*}\left(X ; \mathbb{F}_{p}\right)$ is bounded below and finite in each degree, or the spectral sequence collapses at a finite stage, then the spectral sequence is strongly convergent.

Proposition 7.2 There is a natural spectral sequence

$$
E_{* *}^{2}=H_{*}\left(\mathbb{T} ; H_{*}\left(X ; \mathbb{F}_{p}\right)\right)=P\left(y^{-1}\right) \otimes H_{*}\left(X ; \mathbb{F}_{p}\right)
$$

with $y^{-1}$ in bidegree $(2,0)$, which is strongly convergent to $H_{*}\left(X_{h \mathbb{T}} ; \mathbb{F}_{p}\right)$. We call this the homological homotopy orbit spectral sequence. (Note that for $X_{h \mathbb{T}}$ the continuous homology is the same as the ordinary homology.)

Further, the middle and right hand maps of the (homotopy) norm cofiber sequence

$$
\Sigma X_{h \mathbb{T}} \stackrel{N}{\longrightarrow} X^{h \mathbb{T}} \rightarrow X^{t \mathbb{T}} \rightarrow \Sigma^{2} X_{h \mathbb{T}}
$$

induce the homomorphisms of $E^{2}$-terms given by tensoring $H_{*}\left(X ; \mathbb{F}_{p}\right)$ with the short exact sequence of $P(y)$-modules

$$
0 \rightarrow P(y) \rightarrow P\left(y, y^{-1}\right) \rightarrow \Sigma^{2} P\left(y^{-1}\right) \rightarrow 0 .
$$


Thus the homological Tate spectral sequence is a full-plane spectral sequence whose $E^{2}$-term is obtained by continuing the $y$-periodicity in the homological homotopy fixed point spectral sequence into the right half-plane, and the homological homotopy orbit spectral sequence (shifted 2 degrees to the right from Proposition (7.2) has the quotient of these as its $E^{2}$-term.

Proposition 4.2 and Theorem 5.1 apply equally well to all three spectral sequences. For details, see the thesis of Lunøe-Nielsen [L-N].

Second, we could also consider these three spectral sequences for the action of a finite cyclic subgroup $C$ of $\mathbb{T}$. For example, there is the homological Tate spectral sequence

$$
\widehat{E}_{* *}^{2}=\widehat{H}^{-*}\left(C ; H_{*}\left(X ; \mathbb{F}_{p}\right)\right)
$$

converging conditionally to $H_{*}^{c}\left(X^{t C} ; \mathbb{F}_{p}\right)$. The analogue of Lemma 4.3 still holds, so that there are isomorphisms

$$
\widehat{E}_{* *}^{r} \cong \widehat{H}^{-*}\left(C ; \mathbb{F}_{p}\right) \otimes \widehat{E}_{0, *}^{r}
$$

for all $r \geq 2$ (and now $y$ is invertible, so there is no $y$-torsion), and all differentials are determined by those originating on the vertical axis $\widehat{E}_{0, *}^{r}$. In turn, the latter differentials are determined by those in the $\mathbb{T}$-equivariant case, by naturality with respect to the restriction map $X^{t \mathbb{T}} \rightarrow X^{t C}$. Therefore the collapse results in Theorem [5.1] also hold in these cases. See [L-N] for more details.

These latter spectral sequences, for finite subgroups $C \subset \mathbb{T}$, are essential in the analysis of the topological model $T F(B)$ for the negative cyclic homology of $B$, and the topological cyclic homology $T C(B)$.

Though the differentials here allow us to determine $E_{* *}^{\infty}$ in the cases of interest (see Section 6 ), there are still $A_{*}$-comodule extensions hidden by the filtration. These are of course of critical importance for the analysis of the Adams spectral sequence (1.3). A more elaborate study of the geometry of the universal examples used in Section 5 allows these to be recovered. This too can be found in [L-N].

Finally, it is interesting to compare the formulas for differentials here to analogous results in other spectral sequences. The first to be considered was the Adams spectral sequence, where the results are due to Kahn Ka70, Milgram [Mi72, Mäkinen [Mak73] and the first author [BMMS86, Ch. VI]. For simplicity, let us assume $p=2$ in this discussion, as there are several cases to be considered at odd primes ([BMMS86, VI.1.1]). Suppose that $x$ is in the $E_{r}$-term of the Adams spectral sequence

$$
E_{2}^{* *}=\operatorname{Ext}_{A}^{* *}\left(H^{*}\left(R ; \mathbb{F}_{2}\right), \mathbb{F}_{2}\right) \Longrightarrow \pi_{*}(R)_{2}^{\wedge},
$$


where $R$ is a commutative $S$-algebra. The commutative $S$-algebra structure of $R$ induces Steenrod operations in the $E_{2}$-term of the Adams spectral sequence, which are the analog in this situation of the Dyer-Lashof operations in $H_{*}\left(R ; \mathbb{F}_{2}\right)$. (In fact, under the Hurewicz homomorphism, they map to the Dyer-Lashof operations.) Then, in most cases we have

$$
d_{*}\left(S q^{j} x\right)=S q^{j} d_{r}(x) \dot{+} a S q^{j-v} x,
$$

where $A \dot{+} B$ denotes whichever of $A$ or $B$ is in the lower filtration, or their sum, if they are in the same filtration. The subscript in $d_{*}$ is then the difference in filtrations between the right and left hand sides. In this formula, $a$ is an infinite cycle in the Adams spectral sequence for the homotopy groups of spheres, and $a$ and $v$ are determined by $j$ and the degree of $x$. When the first half of the right hand side dominates we have

$$
d_{2 r-1}\left(S q^{j} x\right)=S q^{j} d_{r}(x),
$$

and this formula resembles the formula

$$
d^{2 r}\left(\beta^{\epsilon} Q^{i}(x)\right)=\beta^{\epsilon} Q^{i}\left(d^{2 r}(x)\right)
$$

of Proposition 4.2. in that both essentially say that the relevant differential commutes with the Dyer-Lashof operations. The fact that the length of the differential increases from $r$ to $(2 r-1)$ when we apply the squaring operation in the Adams spectral sequence reflects the difference between the homotopy fixed point filtration and the Adams filtration, and the way in which they interact with the extended powers. A more extreme difference occurs when the second term $a S q^{j-v} x$ is involved. In the homological homotopy fixed point spectral sequence this term disappears, essentially because the element $a \in \pi_{*} S$ is mapped to 0 by the Hurewicz homomorphism. Homotopical homotopy fixed point spectral sequences, as in AuR02, will have differential formulas with two parts, as in the Adams spectral sequence. Such two part formulas for differentials reflect universally hidden extensions in the following sense.

The differential (17.1) arises from decomposing the boundary of the cell on which $S q^{j} x$ is defined into two pieces. One of the pieces carries $S q^{j} d_{r}(x)$ and the other carries $a S q^{j-v} x$. The half that lies in the lower filtration is killed by the differential (7.1), and therefore appears to be 0 in the associated graded $E_{\infty}$ term. However, the geometry of the situation shows that it is actually equal to the half of the formula that lies in the higher filtration, modulo still higher filtrations. Thus we have a universally hidden extension, that is, an expression which is 0 in the associated graded, by virtue of being equal to an expression which lies in a higher filtration. We should expect this sort of phenomenon to occur in homotopical homotopy fixed point spectral sequences. 
Finally, Theorem 5.1 seems to be particular to the homological homotopy fixed point spectral sequence. Certainly the Adams spectral sequence seems to have no analog of this extreme cutoff, in which certain terms die at $E^{r}$ and the remaining terms live to $E^{\infty}$.

\section{References}

[AGM85] J F Adams, J H Gunawardena and $\mathbf{H}$ Miller, The Segal conjecture for elementary abelian p-groups, Topology 24 (1985) 435-460 MathReview

[AnR] V Angeltveit and J Rognes, Hopf algebra structure on topological Hochschild homology, Algebraic and Geometric Topology (to appear)

[AuR02] Ch Ausoni and J Rognes, Algebraic K-theory of topological K-theory, Acta Math. 188 (2002) 1-39 MathReview

[BJ02] A Baker and A Jeanneret, Brave new Hopf algebroids and extensions of $M$ U-algebras, Homology Homotopy Appl. 4 (2002) 163-173 MathReview

[Bo99] J M Boardman, Conditionally convergent spectral sequences, in Homotopy invariant algebraic structures, American Mathematical Society, Contemp. Math. 239 (1999) 49-84 MathReview

[BHM93] M Bökstedt, W C Hsiang and I Madsen, The cyclotomic trace and algebraic $K$-theory of spaces, Invent. Math. 111 (1993) 465-539 MathReview

[BG95] R Bruner and J Greenlees, The Bredon-Löffler Conjecture, Exp. Math. 4 (1995) 289-297 MathReview

[BMMS86] R R Bruner, J P May, J E McClure and $\mathbf{M}$ Steinberger, $H_{\infty}$ ring spectra and their applications, Lecture Notes in Mathematics 1176, Springer-Verlag (1986) MathReview

[Ca84] G Carlsson, Equivariant stable homotopy and Segal's Burnside ring conjecture, Ann. of Math. (2) 120 (1984) 189-224 MathReview

[CE56] H Cartan and S Eilenberg, Homological Algebra, Princeton Mathematical Series 19 Princeton University Press (1956) MathReview

[CMP87] J Caruso, J P May and S B Priddy, The Segal conjecture for elementary p-groups. II. p-adic completion in equivariant cohomology, Topology 26 (1987) 413-433 MathReview

[CS] R Cohen and Ch Schlichtkrull, Topological Hochschild homology of Thom spectra, (in preparation)

[Du97] BI Dundas, Relative $K$-theory and topological cyclic homology, Acta Math. 179 (1997) 223-242 MathReview

[EKMM97] A D Elmendorf, I Kriz, M A Mandell and J P May, Rings, modules, and algebras in stable homotopy theory. With an appendix by $M$ Cole, Mathematical Surveys and Monographs 47 American Mathematical Society (1997) MathReview

[GM95] J P C Greenlees and J P May, Generalized Tate cohomology, Mem. Am. Math. Soc. 543 (1995) MathReview 
[HM03] L Hesselholt and I Madsen, On the $K$-theory of local fields, Ann. of Math. (2) 158 (2003) 1-113 MathReview

[HSS00] M Hovey, B Shipley and J Smith, Symmetric spectra, J. Am. Math. Soc. 13 (2000) 149-208 MathReview

[Ka70] D S Kahn, Cup-i products and the Adams spectral sequence, Topology 9 (1970) 1-9 MathReview

[LMS86] L G Lewis Jr, J P May and M Steinberger, Equivariant stable homotopy theory. With contributions by JE McClure, Lecture Notes in Mathematics 1213, Springer-Verlag (1986) MathReview

[LDMA80] W H Lin, D M Davis, ME Mahowald and J F Adams, Calculation of Lin's Ext groups, Math. Proc. Camb. Philos. Soc. 87 (1980) 459-469 MathReview

[L-N] S Lunøe-Nielsen, The Segal conjecture for topological Hochschild homology of commutative $S$-algebras, University of Oslo Ph.D. thesis (2005)

[Mc01] J McCleary, A user's guide to spectral sequences, 2nd ed. Cambridge Studies in Advanced Mathematics 58, Cambridge University Press (2001) MathReview

[MM02] M A Mandell and J P May, Equivariant orthogonal spectra and $S$ modules, Mem. Am. Math. Soc. 159 (2002) MathReview

[MMSS01] M A Mandell, J P May, S Schwede and B Shipley, Model categories of diagram spectra, Proc. Lond. Math. Soc., III. Ser. 82 (2001) 441-512 MathReview

[Mak73] J Mäkinen, Boundary formulae for reduced powers in the Adams spectral sequence, Ann. Acad. Sci. Fenn., Ser. A I 562 (1973) 42 pp. MathReview

[Ma54] W S Massey, Products in exact couples, Ann. of Math. (2) 59 (1954) 558569 MathReview

[MS93] J E McClure and R E Staffeldt, On the topological Hochschild homology of bu. I, Am. J. Math. 115 (1993) 1-45 MathReview

[Mi72] R J Milgram, Group representations and the Adams spectral sequence, Pacific J Math. 41 (1972) 157-182 MathReview

[Mi58] J Milnor, The Steenrod algebra and its dual, Ann. of Math. (2) 67 (1958) 150-171 MathReview

[Ro98] J Rognes, Trace maps from the algebraic $K$-theory of the integers (after Marcel Bökstedt), J. Pure Appl. Algebra 125 (1998) 277-286 MathReview

[Ro02] J Rognes, Two-primary algebraic K-theory of pointed spaces, Topology 41 (2002) 873-926 MathReview

[Ro03] J Rognes The smooth Whitehead spectrum of a point at odd regular primes, Geometry and Topology 7 (2003) 155-184 MathReview

Department of Mathematics, Wayne State University, Detroit, MI 48202, USA

Department of Mathematics, University of Oslo, NO-0316 Oslo, Norway

Email: rrb@math.wayne.edu, rognes@math.uio.no

Received: 2 June 2004 Revised: 3 June 2005 